\title{
Conduction Band-Edge States Associated With the Removal of d-State Degeneracies by the Jahn-Teller Effect
}

\author{
Gerald Lucovsky, C. C. Fulton, Y. Zhang, Y. Zou, J. Luning, L. F. Edge, J. L. Whitten, R. J. Nemanich, H. Ade, \\ D. G. Schlom, V. V. Afanase'v, A. Stesmans, S. Zollner, D. Triyoso, and B. R. Rogers
}

Invited Paper

\begin{abstract}
X-ray absorption spectroscopy (XAS) is used to study band edge electronic structure of high- $\kappa$ transition metal (TM) and trivalent lanthanide rare earth $(\mathrm{RE})$ oxide gate dielectrics. The lowest conduction band $\mathrm{d}^{*}$-states in $\mathrm{TiO}_{2}, \mathrm{ZrO}_{2}$ and $\mathrm{HfO}_{2}$ are correlated with: 1) features in the $O K_{1}$ edge, and 2) transitions from occupied Ti 2p, $\mathrm{Zr} 3 \mathrm{p}$ and $\mathrm{Hf} 4 \mathrm{p}$ states to empty Ti 3d-, $\mathrm{Zr}$ 4d-, and Hf 5d-states, respectively. The relative energies of d-state features indicate that the respective optical bandgaps, $\mathrm{E}_{\text {opt }}$ (or equivalently, $\mathrm{E}_{\mathrm{g}}$ ), and conduction band offset energy with respect to $\mathrm{Si}$, $E_{B}$, scale monotonically with the d-state energies of the TM/RE atoms. The multiplicity of d-state features in the $T i L_{2,3}$ spectrum of $\mathrm{TiO}_{2}$, and in the derivative of the $\mathrm{O} \mathrm{K}_{1}$ spectra for $\mathrm{ZrO}_{2}$ and $\mathrm{HfO}_{2}$ indicate a removal of d-state degeneracies that results from a static Jahn-Teller effect in these nanocrystalline thin film oxides. Similar removals of d-state degeneracies are demonstrated for complex TM/RE oxides including $\mathrm{Zr}$ and $\mathrm{Hf}$ titanates, and $\mathrm{La}$, Gd and Dy scandates. Analysis of XAS and band edge spectra indicate an additional band edge state that is assigned Jahn-Teller distortions at internal grain boundaries. These band edges defect states are electronically active in photoconductivity (PC), internal photoemission (IPE), and act as bulk traps in metal oxide semiconductor (MOS) devices, contributing to asymmetries in tunneling and Frenkel-Poole transport that have important consequences for performance and reliability in advanced Si devices.
\end{abstract}

Index Terms-Complex oxides, conduction band edge states, d-state degeneracy, high- $\kappa$ dielectrics, Jahn-Teller splittings, photoconductivity, spectroscopic ellipsometry, x-ray absorption spectroscopy.

Manuscript received September 28, 2004; revised January 11, 2005. The work of G. Lucovsky was supported by the Office of Naval Research (ONR), the Air Force Office of Scientific Research (AFOSR), the Semiconductor Research Corporation (SRC), and the SRC/International SEMATECH Front End Processes (FEP) Center.

G. Lucovsky, C. C. Fulton, Y. Zhang, Y. Zou, R. J. Nemanich and H. Ade are with the Department of Physics, North Carolina State University, Raleigh, NC 27695 USA (e-mail: lucovsky@ncsu.edu).

J. Luning is with the Stanford Synchrotron Radiation Laboratories, Menlo Park, CA 94025 USA.

L. F. Edge and D. G. Schlom are with the Department of Materials Science and Engineering, Pennsylvania State University, State College, PA 16801 USA.

J. L. Whitten is with the Department of Chemistry, North Carolina State University, Raleigh, NC 27695 USA.

V. V. Afanase'v and A. Stesmans are with the Department of Physics, University of Leuven, Leuven, Belgium.

S. Zollner and D. Triyoso are with Freescale Semiconductor, Inc., Tempe, AZ 85284 USA.

B. R. Rogers is with the Department of Chemical Engineering, Vanderbilt University, Nashville, TN 37235 USA.

Digital Object Identifier 10.1109/TDMR.2005.845804

\section{INTRODUCTION}

$\mathbf{T}$ HERE has been a search for alternative dielectrics with significantly increased dielectric constants, $\kappa$, with respect to $\mathrm{SiO}_{2}$ to reduce direct tunneling in field effect transistors (FETs) with the equivalent oxide thickness, EOT $<1.5 \mathrm{~nm}$. These substitutions for $\mathrm{SiO}_{2}$ would allow relative increases in physical thickness proportional to $\kappa$ for a given gate dielectric capacitance, thereby providing the possibility for significantly reducing direct tunneling. However, increases in $\kappa$ are generally accompanied by decreases in the bandgap, $\mathrm{E}_{\mathrm{opt}}$, the conduction band offset energy, $\mathrm{E}_{\mathrm{B}}$, and the effective electron tunneling mass, $\mathrm{m}_{\mathrm{eff}}^{*}$, so that reductions in direct tunneling cannot be determined from increases in $\kappa$ and physical thickness alone. These tradeoffs are quantified in a tunnelling figure of merit, $\Phi_{\mathrm{m}}$, given by

$$
\Phi_{\mathrm{m}}=\mathrm{k}\left[\mathrm{E}_{\mathrm{B}} \cdot \mathrm{m}_{\mathrm{eff}}^{*}\right]^{0.5}
$$

This paper combines $\mathrm{x}$-ray absorption spectroscopy (XAS) with other band edge spectroscopic studies to provide in information relative to the band edge states that determine $\mathrm{E}_{\mathrm{opt}}$ and $\mathrm{E}_{\mathrm{B}}$. As such, the reported research results address intrinsic bonding effects that significantly limit integration of high- $\kappa$ elemental oxides, e.g., $\mathrm{HfO}_{2}$, into gate stacks for aggressively scaled Si complementary metal-oxide-semiconductor (CMOS) devices. These same limitations are alo studied in complex oxides comprised of transition metal (TM) oxides in combination with: 1) other TM oxides, such as in $\mathrm{HfTiO}_{4}$ or $\left.\left(\mathrm{HfO}_{2}\right)_{1.0}\left(\mathrm{TiO}_{2}\right)_{1.0} ; 2\right)$ trivalent lanthanide series rare earth (RE) oxides, such as $\mathrm{LaScO}_{3}$ or $\left(\mathrm{La}_{2} \mathrm{O}_{3}\right)_{0.5}\left(\mathrm{Sc}_{2} \mathrm{O}_{3}\right)_{0.5}$; as well as 3) La and other RE/TM aluminates and silicates. One limitation relates to energies of the lowest d-state derived conduction bands relative to the conduction band edge of crystalline $\mathrm{Si}$. This is designated as the conduction band offset energy, $\mathrm{E}_{\mathrm{B}}$, which defines the tunneling barrier at Si/high- $\kappa$ dielectric interfaces. The high- $\kappa$ TM metal oxides with the largest dielectric constants, $\mathrm{k}$, relative to $\mathrm{SiO}_{2}$, e.g., $\mathrm{TiO}_{2}$, $\mathrm{Ta}_{2} \mathrm{O}_{5}$, etc., have the smallest optical bandgaps, $\mathrm{E}_{\mathrm{opt}}$, and conduction band offset energies [1], [2]. This paper demonstrates that calculated conduction band offset energies scale monotonically with calculated and/or measured optical bandgaps. 


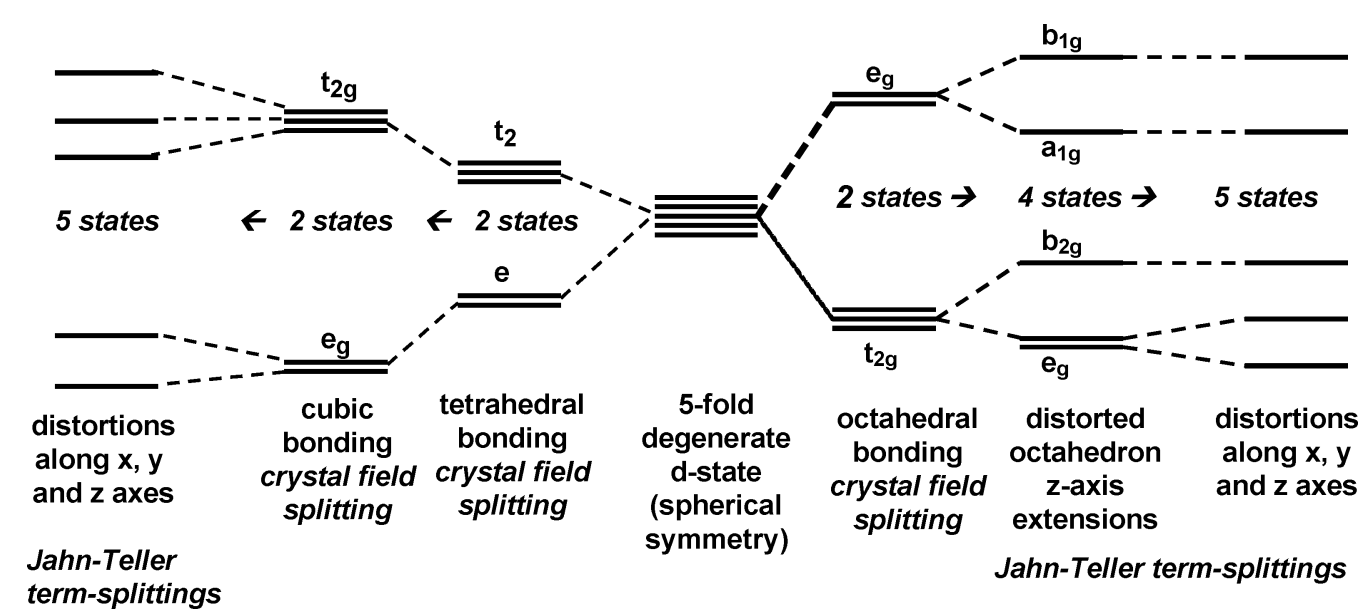

Fig. 1. Schematic representation of the transition from spherically symmetric d-state bonding, to crystal-field and Jahn-Teller degeneracy reductions.

The high- $\kappa$ elemental oxides with the highest values of $\kappa$ have conduction band offset energies that are too small, $1.0 \mathrm{eV}$, to satisfy projected tunneling leakage limits for scaled devices with EOT below $1.5 \mathrm{~nm}$. One possible solution is to introduce these TM oxides into complex binary oxides, and increase conduction band offset energies through a coupling of the constituent oxide TM/RE atom d-states produced by bonding to a common O-atom. In contrast and based on $\mathrm{O} \mathrm{K}_{1}$ spectra of the constituent elemental oxides, the spectroscopic studies of this paper have identified more than two d-states in the complex oxides; however, their energies in $\mathrm{O} \mathrm{K}_{1}$ edge spectra suggest at most weak coupling between the $\mathrm{d}$-states of the constituent TM atoms. On the other hand, the multiplicity of d-state features in the Ti and $\mathrm{Sc} \mathrm{L}_{2,3}$ edge spectra has suggested an alternative mechanism for the multiplicity of d-states found in the complex oxides as well.

This alternative mechanism derives from Jahn-Teller distortions that remove the degeneracies of the constituent atom valence and conduction band d-states through localized bonding distortions that are present in nanocrystalline, polycrystalline, and single crystalline complex oxides [3], [4]. These term-split $\mathrm{d}$-states have been identified by $\mathrm{x}$-ray absorption spectroscopy (XAS), as well as optical and uv absorption and ellipsometry. The removal of d-state degeneracies through bonding distortions that reduce local symmetry is the origin of large dynamic infrared (IR) charges, which lower frequencies of the IR active vibrations that contribute to the high values of $\kappa$ in $\mathrm{TiO}_{2}, \mathrm{Ta}_{2} \mathrm{O}_{5}$ and $\mathrm{Sc}_{2} \mathrm{O}_{3}$ [4]. This papers will demonstrate that Jahn-Teller term-split states, generated by larger bonding distortions at internal boundaries present in nanocrystalline dielectrics, generate localized states below the intrinsic conduction band edge $\mathrm{d}$-states from the nanocrystallite grains, and that these localized states are active as interface and bulk traps in Si MOS devices, contributing to asymmetric shifts in field effect transistor (FET) threshold voltages, $V_{\mathrm{t}}$, that degrade CMOS circuit operation.

Before addressing the XAS spectra, it is important to trace the evolution of the TM/RE d-states from the free atoms to the bonding environment of the oxides addressed in this review paper. The five-fold degeneracies of TM/RE d-states in an atom are partially removed by spin-orbit (S-O) splittings. These increase with increasing atomic number, $\mathrm{z}$, and decreasing principal quantum number, $n$. For example, relatively deep core states such as the 3 $\mathrm{d}$-state in $\mathrm{Hf}$ at $\sim 1700 \mathrm{eV}$, have a S-O splitting of $\sim 50 \mathrm{eV}$ [5]. The $\mathrm{S}$-O splitting in the valence $5 \mathrm{~d}$-states in $\mathrm{Hf}$ is reduced to $\sim 0.32$ $\mathrm{eV}$ [5], and is the largest of the TM/RE elements addressed in this paper. For example, the S-O splittings of the $\mathrm{Zr} 4 \mathrm{~d}$, and $\mathrm{Ti} 3 \mathrm{~d}$ states are $0.10 \mathrm{eV}$ and $0.04 \mathrm{eV}$, respectively, and lower than the Hf $5 \mathrm{~d} \mathrm{S-O}$ splitting. In contrast crystal-field (C-F) splittings for symmetric six-fold and eight-fold bonding environments, typical of TM/RE atoms in elemental and complex oxides, are generally 2 to $3 \mathrm{eV}$. S-O splittings always result in lower energies for the doubly degenerate $\mathrm{d}_{3 / 2}$ as compared to the triply degenerate $\mathrm{d}_{5 / 2}$ atomic states, whereas C-F splittings are qualitatively different with the lowest conduction band d-states for six-fold coordination being derived from the $\mathrm{d}_{5 / 2}$ atomic state, or equivalently, the triply degenerate $\mathrm{T}_{2 \mathrm{~g}}$ state, and for eight-fold coordination from the $\mathrm{d}_{3 / 2}$ atomic state, or equivalently, the doubly degenerate $\mathrm{E}_{\mathrm{g}}$ state. Distortions in the six- or eight-fold coordinated bonding of the TM/RE atoms, that derive from the static J-Teffect, either partially, or totally, remove the degeneracies of the $\mathrm{E}_{\mathrm{g}}$ and $\mathrm{T}_{2 \mathrm{~g}}$ states, resulting in at most five distinct d-states. The J-T term splittings depend on the magnitude and symmetry of the distortions, and are typically about $0.5-0.7 \mathrm{eV}$. It is also common practice to characterize the static J-T effect as a C-F effect resulting from a distorted local bonding geometry. The $J$-T designation gives additional insight into the mechanism that drives the degeneracy removal.

As noted above, the partial or complete removal of the $\mathrm{E}_{\mathrm{g}}$ and $\mathrm{T}_{2 \mathrm{~g}}$ degeneracies is determined by the nature of the distortion. For example, a symmetric $z$ axis distortion of the octahedral bonding environment of a Ti atom (see Fig. 1), completely removes the degeneracy of the higher lying $\mathrm{E}_{\mathrm{g}}$ states, but only partially removes the degeneracy of the lower lying $\mathrm{T}_{2 \mathrm{~g}}$ state, whereas, a symmetric $z$ axis distortion, coupled with the conversion of the square in the $x-y$ plane to rectangle completely removes the degeneracies of both the $E_{g}$ and $T_{2 g}$ states yielding five distinct d-states. Fig. 1 also includes a schematic representation for the S-O, C-F and J-T effects for four-fold coordinated atom in a tetrahedral, as well as eight-fold coordinated TM atoms, e.g., $\mathrm{Zr}$ or Hf, in cubic and distorted environments. This diagram provides a road-map for understanding the spectral features identified and discussed in the next few sections of this review. Since the d-state S-O splittings are generally small 
relative to the C-F splittings, and the changes in these due to J-T effects, the $\mathrm{S}-\mathrm{O}$ will be neglected in the discussions that follow.

\section{EXPERIMENTAL PROCEDURES}

XAS measurements were performed at the National Synchrotron Light Source (NSLS) at the Brookhaven National Laboratory (BNL), and at the Stanford Synchrotron Radiation Laboratories (SSRL) at the Stanford Linear Accelerator Center (SLAC) using total photo-electron yield to determine the relative absorption strength of the spectral features associated with transitions from TM, RE, and O-atom core level states to empty conduction band-states [6]. Thin film samples of $\mathrm{ZrO}_{2}$ and $\mathrm{HfO}_{2}$ and their respective silicate alloys were prepared by remote plasma enhanced chemical vapor deposition (RPECVD) [7]. $\mathrm{TiO}_{2}$ and $\left(\mathrm{Hf}(\mathrm{Zr}) \mathrm{O}_{2}\right)_{\mathrm{x}}\left(\mathrm{TiO}_{2}\right)_{1-\mathrm{x}}$ alloy thin films were prepared by physical deposition of $\mathrm{Ti}$ and $\mathrm{Hf}(\mathrm{Zr})$ atoms, followed by in situ plasma-assisted oxidation, and by reactive evaporation in an ultrahigh vacuum system in both instances using e-beam sources for the TM metals [8]. The growth of $\mathrm{GdScO}_{3}$ and $\mathrm{DyScO}_{3}$ single crystals has been discussed in [9]. Thin films of $\mathrm{LaAlO}_{3}$, and $\left(\mathrm{La}(\mathrm{Dy}, \mathrm{Gd})_{2} \mathrm{O}_{3}\right)_{1-\mathrm{x}}\left(\mathrm{Sc}_{2} \mathrm{O}_{3}\right)_{\mathrm{x}}$, were also grown by reactive evaporation in an ultrahigh vacuum deposition system using effusion cell sources for the TM and RE atoms [10].

\section{X-RAY AbSORPTION SPECTROSCOPY STUdiES OF TRANSITION METAL-OXIDE FILMS}

\section{A. Differences Between Intra- and Inter-Atomic Spectra}

Fig. 2(a) and (b) presents $\mathrm{Zr} \mathrm{M} \mathrm{M}_{2,3}$ and $\mathrm{O} \mathrm{K}_{1}$ spectra for thin film nanocrystalline $\mathrm{ZrO}_{2}$ [6]. The $\mathrm{Zr} \mathrm{M}_{2,3}$ spectrum in Fig. 2(a) is effectively an intra-atomic spectrum in which dipole-allowed transitions from occupied $\mathrm{Zr}$ core level, spin-orbit split 3p-states $\left(3 \mathrm{p}_{1 / 2}\right.$ and $\left.3 \mathrm{p}_{3 / 2}\right)$ terminate in empty $\mathrm{Zr} 4 \mathrm{~d}$ and $5 \mathrm{~s}$ states that lie well above the top of the valence band edge, $\sim 5-6 \mathrm{eV}$. The energy differences between: 1) the spectral peaks of two 3d-states with triply degenerate $\mathrm{E}_{\mathrm{g}}$ and doubly degenerate $\mathrm{T}_{2 \mathrm{~g}}$ character, $\Delta\left(\mathrm{d}_{1}, \mathrm{~d}_{2}\right)$, and 2$)$ the spectral peak of the first $3 \mathrm{~d}$-state and the $4 \mathrm{~s}$-state $\Delta\left(\mathrm{d}_{1}, \mathrm{~s}\right)$ are in good agreement $( \pm 0.3 \mathrm{eV})$ with ab inito calculations based on small clusters with central $\mathrm{Zr}$ atoms, and two shells of atomic neighbors [11]. The relative intensities of the $4 \mathrm{~d}-$ and $5 \mathrm{~s}-$ features are markedly different, and are consistent with the contributions of the $\mathrm{Zr}$ atomic radial wave functions of the initial and final states to the transition matrix element [12]. The ab initio calculations also provide equally good agreement with respect to the $\mathrm{Zr}$-O bond length, and the spectral peaks of features in the valence band spectrum as determined by UV photoemission spectroscopy [8], [11].

The $\mathrm{ZrO}_{2} \mathrm{O} \mathrm{K}_{1}$ spectrum in Fig. 2(b) is an inter-atomic atomic spectrum with final states reflecting a mixing of $\mathrm{O} 2 \mathrm{p}^{*}$ and $\mathrm{Zr} 4 \mathrm{~d}^{*}-, 5 \mathrm{~s}^{*}$ - and $5 \mathrm{p}^{*}$-states. Agreement with ab initio calculations is approximately $\pm 0.3 \mathrm{eV}$ as well [11].

\section{B. Intra- and Inter-Atomic Spectra for $\mathrm{TiO}_{2}, \mathrm{ZrO}_{2}$ and $\mathrm{HfO}_{2}$}

Qualitatively similar spectra have been obtained for the corresponding TM p-state and $\mathrm{O}$ atom core level absorption spectra in $\mathrm{TiO}_{2}$ and $\mathrm{HfO}_{2}$ [6]. These include the $\mathrm{Ti} \mathrm{L}_{2,3}$ and $\mathrm{Hf} \mathrm{N}_{2,3}$

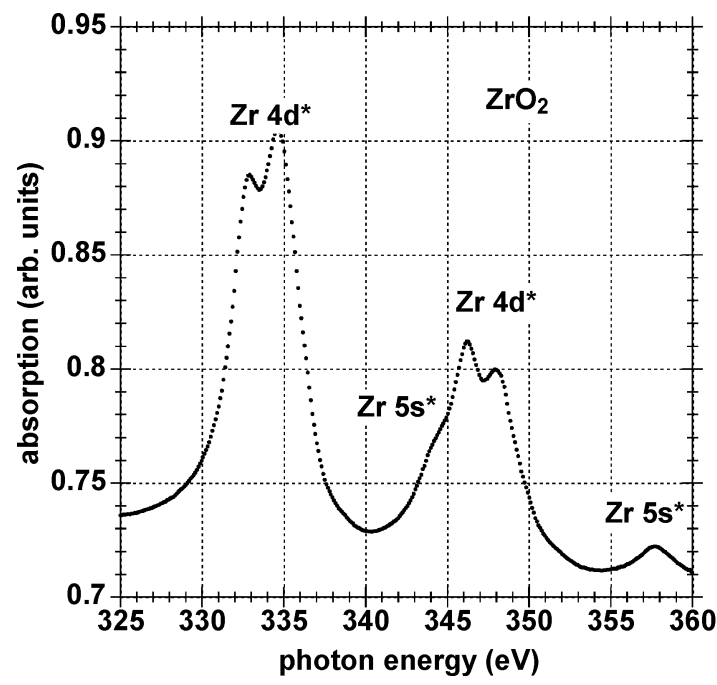

(a)

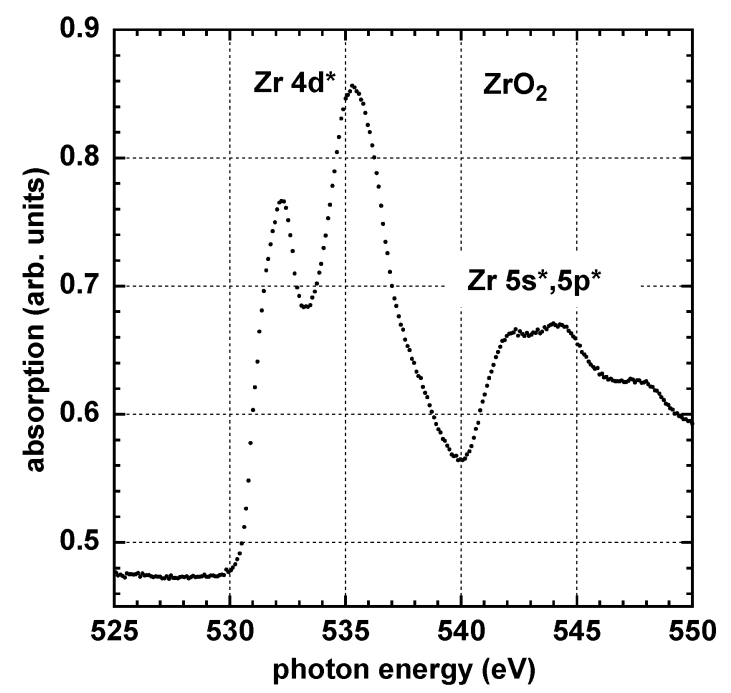

(b)

Fig. 2. Core level x-ray absorption spectra for nanocrystalline $\mathrm{ZrO}_{2}$. (a) $\mathrm{Zr} \mathrm{M}_{2,3}$ spectra for excitation from spin-orbit split $\mathrm{Zr} 3$ p levels to empty $\mathrm{Zr}$ $4 \mathrm{~d}^{*}$ and $5 \mathrm{~s}^{*}$ states, and (b) $\mathrm{O} \mathrm{K} \mathrm{K}_{1}$ spectra for excitation from the $\mathrm{O} 1 \mathrm{~s}$ level to molecular orbital $\mathrm{Zr} 4 \mathrm{~d}^{*}$ and $5 \mathrm{~s}^{*}$ states mixed with $\mathrm{O} 2 \mathrm{p}^{*}$ states.

spectra, as well the respective $\mathrm{TiO}_{2}$ and $\mathrm{HfO}_{2} \mathrm{O} \mathrm{K}_{1}$ spectra. The Ti $\mathrm{L}_{2,3}$ and $\mathrm{O} \mathrm{K}_{1}$ spectra for $\mathrm{TiO}_{2}$ are displayed in Fig. 3(a) and (b), and the $\mathrm{Hf} \mathrm{N}_{2,3}$ and $\mathrm{O} \mathrm{K}_{1}$ spectra for $\mathrm{HfO}_{2}$ are displayed in Fig. 4(a) and (b). Differentiated $\mathrm{O} \mathrm{K}_{1}$ spectra are displayed in Fig. 5(a) and (b) for $\mathrm{ZrO}_{2}$ and $\mathrm{HfO}_{2}$, respectively.

The relative intensities of features that terminate in empty dand s-states, respectively, in $\mathrm{Ti}_{2,3}, \mathrm{Zr} \mathrm{M}_{2,3}$ and $\mathrm{Hf} \mathrm{N}_{2,3}$ spectra are consistent with the intra-atomic character of these transitions. This has been established by comparing the ratios of the integrated absorption of the d-state and s-state features in the respective $\mathrm{L}_{3}, \mathrm{M}_{3}$, and $\mathrm{N}_{3}$ spectra with the transition probabilities for the corresponding Rydberg state transitions [12]. For example, this ratio is $\sim 3.0 \pm 0.3 \times 10^{-2}$ for $2 p$ to $4 \mathrm{~s}$ and $3 \mathrm{~d}$ transitions in $\mathrm{TiO}_{2}$, and increases to $8.9 \pm 0.9 \times 10^{-2}$ for $5 \mathrm{~s}$ to $4 \mathrm{~d}$ transitions in $\mathrm{ZrO}_{2}$ [13]. The relative line-widths of the $\mathrm{d}$ features increase markedly as the atomic number, $\mathrm{Z}$, of the transition metal atom increases. $\mathrm{Z}=22$ for $\mathrm{Ti}, 40$ for $\mathrm{Zr}$ and 72 for 


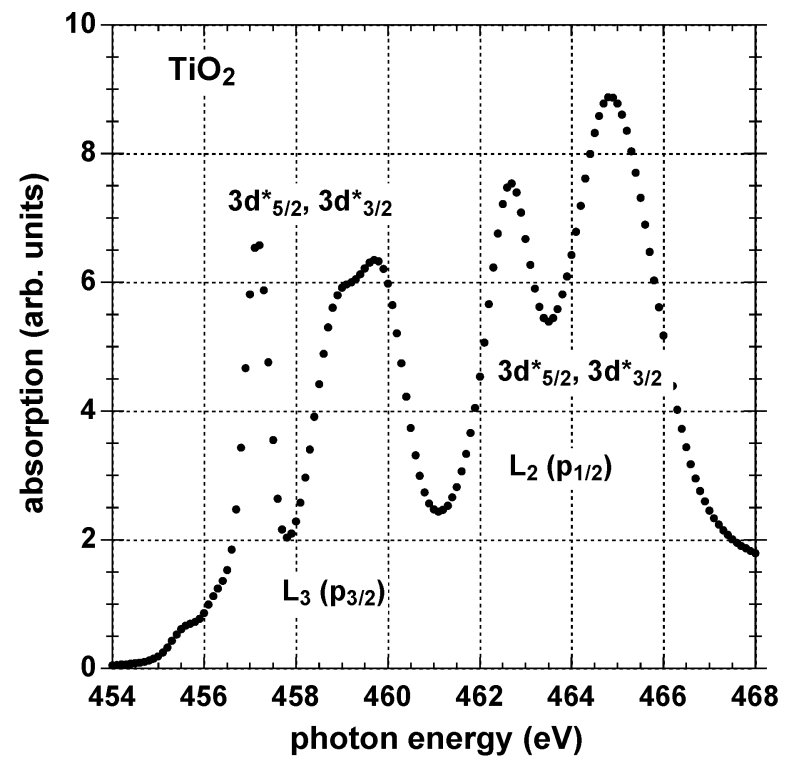

(a)

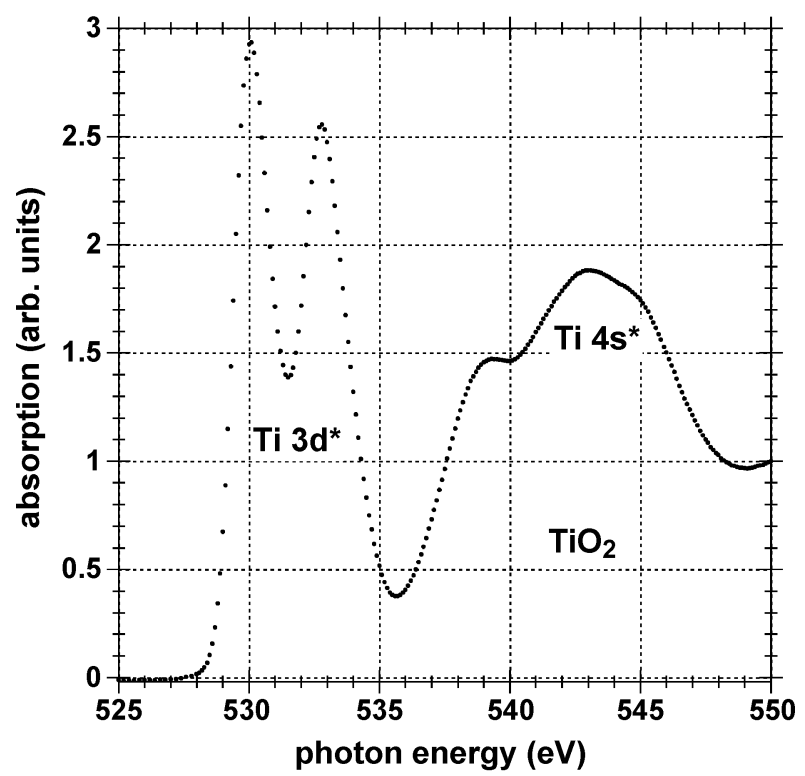

(b)

Fig. 3. Core level $\mathrm{x}$-ray absorption spectra for nanocrystalline $\mathrm{TiO}_{2}$. (a) $\mathrm{Ti}_{2,3}$ and (b) $\mathrm{O} \mathrm{K}_{1}$ spectra.

Hf, and following Slater's empirical rules for intra-atomic transitions, the line-width is expected to increase as $\mathrm{Z}^{\mathrm{n}}$, where $\mathrm{n} \sim 2$ to 3 [13]. This broadening makes impossible to the resolve the doublet character of the $5 \mathrm{~d}$-state in $\mathrm{HfO}_{2}$ in the intra-atomic $\mathrm{N}_{3}$ transition. The $6 \mathrm{~s}$ to $5 \mathrm{~d}$ intensity ratio in the $\mathrm{Hf}_{3}$ spectrum is estimated to be $\sim 0.9$, or about an order of magnitude larger than the corresponding ratio for the $\mathrm{Zr} \mathrm{L}_{3}$ transition, again in good quantitative agreement with intensity ratios obtained for the Rydberg state transition probabilities [12].

The $\Delta\left(\mathrm{d}_{1}, \mathrm{~d}_{2}\right)$ splittings for the dominant spectra features in the $\mathrm{TiO}_{2} \mathrm{Ti} \mathrm{L}_{2,3}$ and the $\mathrm{ZrO}_{2} \mathrm{M}_{2,3}$ spectra are approximately the same, $2.0 \pm 0.3 \mathrm{eV}$ and $2.2 \pm 0.3 \mathrm{eV}$, respectively, as are the corresponding $\Delta\left(\mathrm{d}_{1}, \mathrm{~s}\right)$ splittings of $12.7 \pm 0.3 \mathrm{eV}$ and $11.8 \pm$ $0.3 \mathrm{eV}$. The average $\Delta\left(\mathrm{d}_{1}, \mathrm{~s}\right)$ splitting in $\mathrm{HfO}_{2}$ is about $10 \mathrm{eV}$; if the $\Delta\left(\mathrm{d}_{1}, \mathrm{~d}_{2}\right)$ splitting were about the same as in $\mathrm{TiO}_{2}$ and

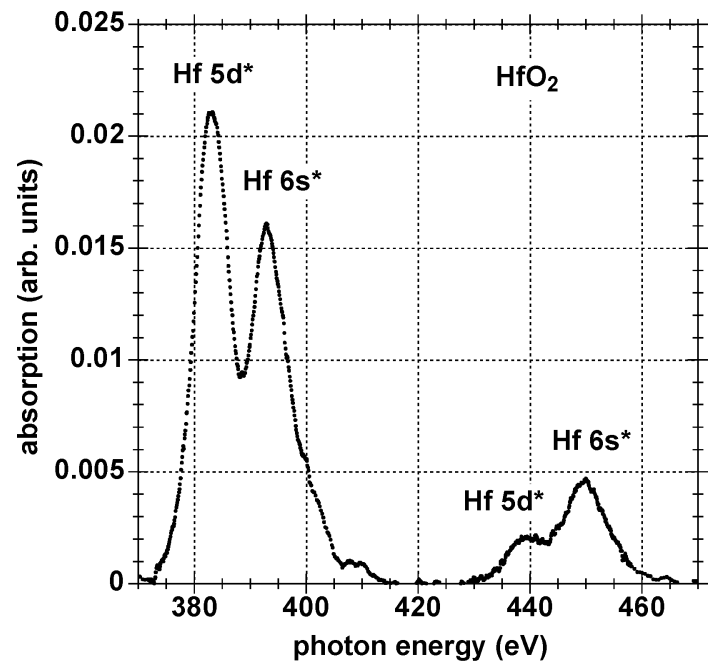

(a)

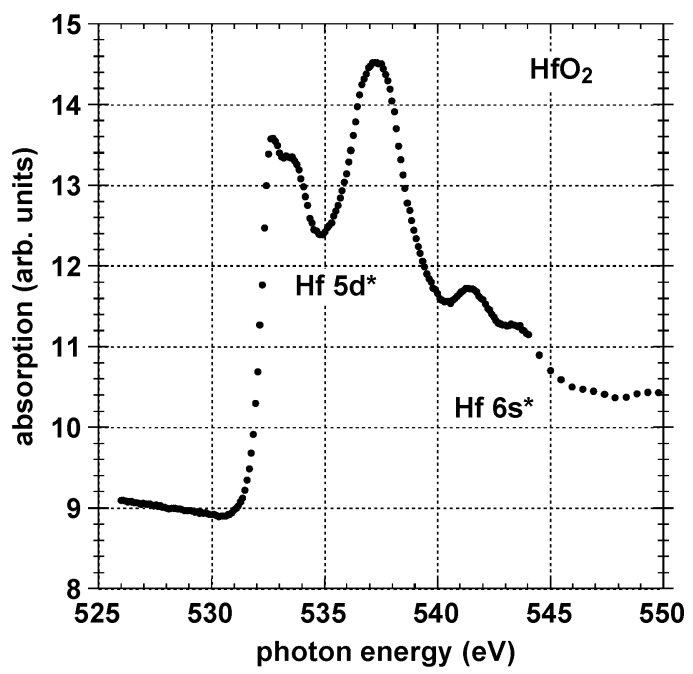

(b)

Fig. 4. Core level x-ray absorption spectra for nanocrystalline $\mathrm{HfO}_{2}$. (a) Hf $\mathrm{N}_{2,3}$ and (b) $\mathrm{O} \mathrm{K}_{1}$ spectra.

$\mathrm{ZrO}_{2}$, then this would increase the estimated splitting to about $11 \mathrm{eV}$. The splittings discussed above are associated with the local bonding symmetries and number of O-atom neighbors of the TM atoms in these thin film TM oxides which are six for $\mathrm{TiO}_{2}$, and eight for $\mathrm{ZrO}_{2}$ and $\mathrm{HfO}_{2}$. Even though the coordinations of these atoms are different these d-state crystal field splittings remain the approximately the same.

The energy differences between the $d_{1}$ and $s$ features these TM oxide spectra do not scale with the differences in the same atomic states in the $3(4,5) \mathrm{d}^{2} 4(5,6) \mathrm{s}^{2}\left(\mathrm{nd}^{2}(\mathrm{n}+1) \mathrm{s}^{2}\right)$ representation appropriate to oxide bonding; $\sim 5.0 \mathrm{eV}$ for $\mathrm{TiO}_{2}, 2.8$ $\mathrm{eV}$ for $\mathrm{ZrO}_{2}$ and $2.4 \mathrm{eV}$ for $\mathrm{HfO}_{2} ; \mathrm{n}$ is the principal quantum number [14]. The $\Delta\left(\mathrm{d}_{1}, \mathrm{~s}\right)$ is approximately the same as in the $\mathrm{L}_{3}, \mathrm{M}_{3}$ and $\mathrm{N}_{3}$ edge spectrum.

The $\mathrm{OK}_{1}$ spectra are inherently inter-atomic in character, and the final states are better described by molecular orbitals (MOs) than empty states as in the $\mathrm{p}$ to $\mathrm{d}$ and $\mathrm{s}$ transitions discussed above [11], [15]. As already noted for $\mathrm{ZrO}_{2}$, these $\mathrm{MO}$ final states mix O $2 \mathrm{p}^{*}$ anti-bonding states with anti-bonding $\mathrm{Zr} 4 \mathrm{~d}^{*}$ 


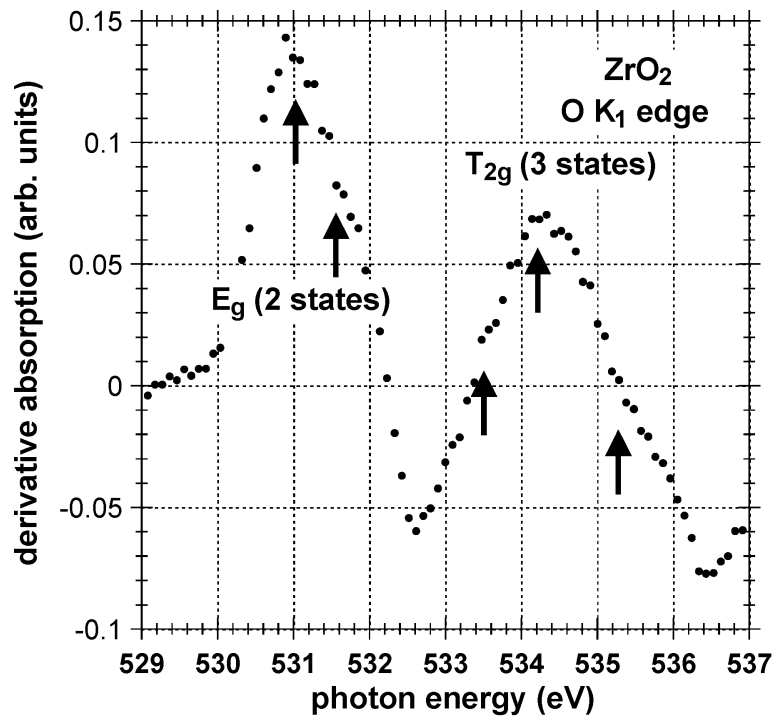

(a)

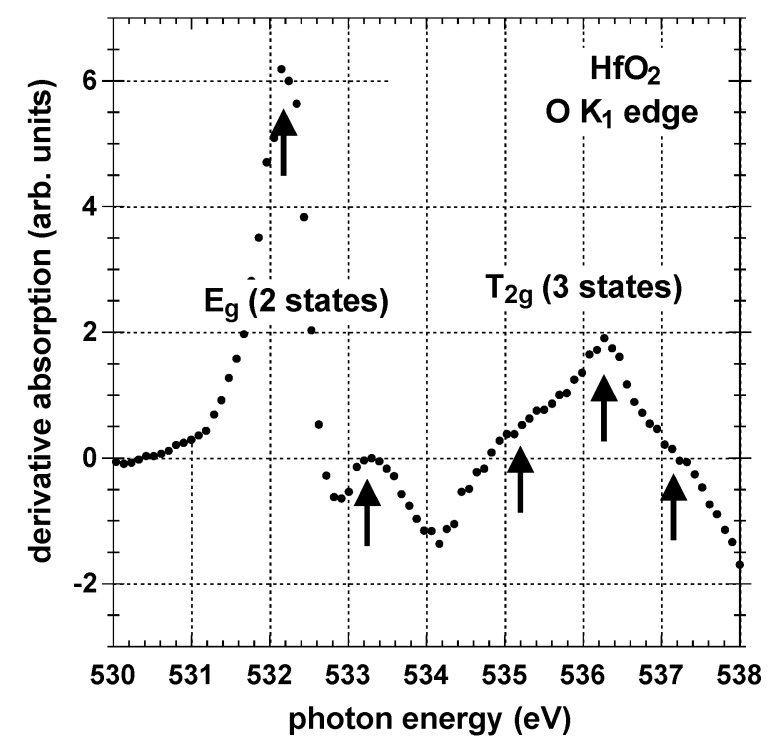

(b)

Fig. 5. Differentiated $\mathrm{O} \mathrm{K}_{1}$ spectra for (a) $\mathrm{ZrO}_{2}$ and (b) $\mathrm{HfO}_{2}$. The markers indicate the multiplicity of $\mathrm{d}^{*}$-state features.

states, with transition matrix elements consistent with the symmetries of the MO initial and final states. These transitions display differences in the relative amplitudes of the doublet $\mathrm{d}^{*}$ features that are related to the six-fold coordination of $\mathrm{Ti}$, and the eight-fold coordination of $\mathrm{Zr}$ and $\mathrm{Hf}$. The $\mathrm{T}_{2 \mathrm{~g}}$ or $\mathrm{d}_{5 / 2}$ triply degenerate states contribute to the $\mathrm{d}_{1}$ feature in $\mathrm{TiO}_{2}$, whereas the $\mathrm{E}_{\mathrm{g}}$ or $\mathrm{d}_{3 / 2}$ doubly degenerate states contribute to the $\mathrm{d}_{1}$ features in $\mathrm{ZrO}_{2}$ and $\mathrm{HfO}_{2}$. The $\Delta\left(\mathrm{d}_{1}^{*}, \mathrm{~d}_{2)}^{*}\right)$ splittings increase from 2.7 $\pm 0.2 \mathrm{eV}$ in $\mathrm{TiO}_{2}$, to in $3.2 \pm 0.2 \mathrm{eV} \mathrm{ZrO}$, and $4.3 \pm 0.2 \mathrm{eV}$ in $\mathrm{HfO}_{2}$, while the $\Delta\left(\mathrm{d}_{1}^{*}, \mathrm{~s}^{*}\right)$ splitting is $8,4 \pm 0.2 \mathrm{eV}$ in $\mathrm{TiO}_{2}$, and approximately the same in $\mathrm{ZrO}_{2}$ and $\mathrm{HfO}_{2}, 10.1 \pm 0.2 \mathrm{eV}$ and $9.6 \pm 0.2 \mathrm{eV}$, respectively.

Equally importantly, the differences in photon energy between the first $\mathrm{d}^{*}$-state spectral peaks in the $\mathrm{OK}_{1}$ spectra of $\mathrm{TiO}_{2}$ at $530.1 \pm 0.2 \mathrm{eV}, \mathrm{ZrO}_{2}$ at $532.2 \pm 0.2 \mathrm{eV}$, and $\mathrm{HfO}_{2}$ at 532. eV, respectively, are the same to within an experimental uncertainty of $\sim 0.3 \mathrm{eV}$ as the differences in the experimentally

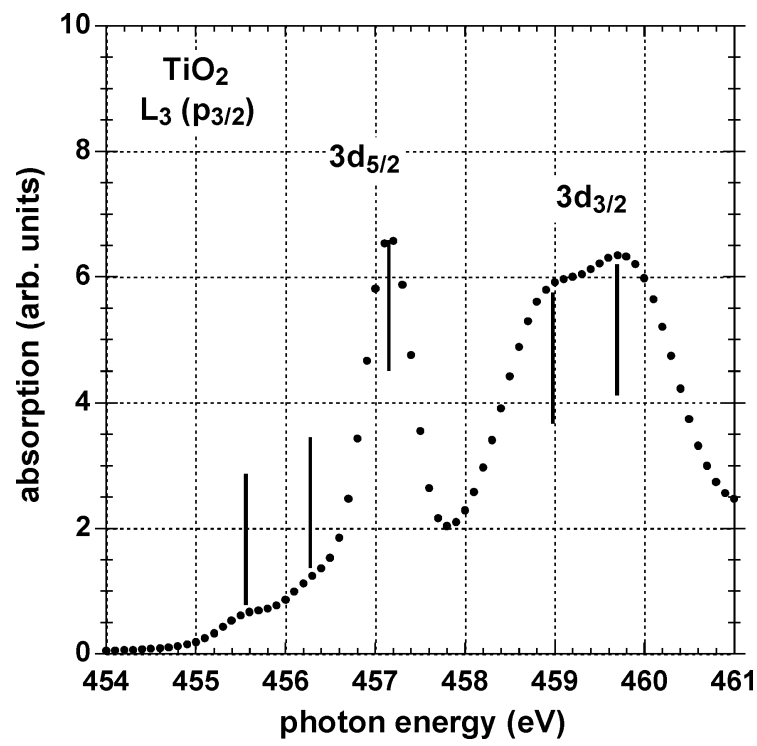

Fig. 6. Expanded $x$ axis plot of the $\mathrm{L}_{3}$ component of the $\mathrm{L}_{2,3}$ spectrum of nanocrystalline $\mathrm{TiO}_{2}$.

determined optical bandgaps, $\mathrm{E}_{\mathrm{g}}, \sim 3.1 \mathrm{eV}$ for $\mathrm{TiO}_{2} \sim 5.6 \mathrm{eV}$ for $\mathrm{ZrO}_{2}$, and $\sim 5.8 \mathrm{eV}$ for $\mathrm{HfO}_{2}$ [1], [2], [6], [15].

The correlation between the energies of the $\mathrm{d}_{1}^{*}$ features in the $\mathrm{O} \mathrm{K}_{1}$ spectra of $\mathrm{TiO}_{2}, \mathrm{ZrO}_{2}$ and $\mathrm{HfO}_{2}$ and the energies of the atomic d-states for the electronic configuration appropriate to oxides, $\mathrm{nd}^{2}(\mathrm{n}+1) \mathrm{s}^{2}$, where $\mathrm{n}=3,4$ and 5 , respectively for $\mathrm{Ti}, \mathrm{Zr}$ and $\mathrm{Hf}$ [6], [14], has important implications for the scaling of these optical bandgaps, $\mathrm{E}_{\text {gap }}$, and conduction band offset energies with respect to $\mathrm{Si}$, defined here as $\mathrm{E}_{\mathrm{B}}$. This will be addressed in Section III-D. It is equally important to correlate features XAS spectra with photoconductivity (PC), internal photoemission (IPE) [16], and band edge dielectric functions and absorption obtained from spectroscopic ellipsometry (SE) studies, and this will be addressed later in this paper for both elemental and complex oxides.

\section{Intra- and Inter-Atomic Spectra for $\mathrm{TiO}_{2}, \mathrm{ZrO}_{2}$ and $\mathrm{HfO}_{2}$}

Fig. 6 displays an expanded view of the $\mathrm{L}_{3}$ spectrum of $\mathrm{TiO}_{2}$. Combined with Fig. 5(a) and (b) which display differentiated spectra of the $\mathrm{O} \mathrm{K}_{1}$ edge spectra of $\mathrm{ZrO}_{2}$ in Fig. 5(a) and $\mathrm{HfO}_{2}$ in Fig. 5(b), these three spectra clearly indicate a multiplicity of five for the d-state features which is greater than what is expected from the local bonding symmetry crystal field splitting which predicts two features corresponding to one doubly degenerate and one triply degenerate state. To better understand the increased d-state multiplicity, the evolution the removal of d-state degeneracies with changes in the local bonding environments of the TM atoms in TM oxides has been presented in Fig. 1. This approach will also be useful in understanding the spectra of more complex oxides, comprised of TM oxides in combination with: 1) other TM oxides as in $\mathrm{HfZiO}_{4}$;2) lanthanide series rare earth (RE) oxides as in $\mathrm{GdScO}_{3}$; and 3) nontransition metal oxides as $\mathrm{LaAlO}_{3}$.

There are no symmetry driven splittings of TM/RE d-states in a spherically symmetric environment of nearest neighbor oxygen atoms, e.g., for a coordination number of twelve as in 


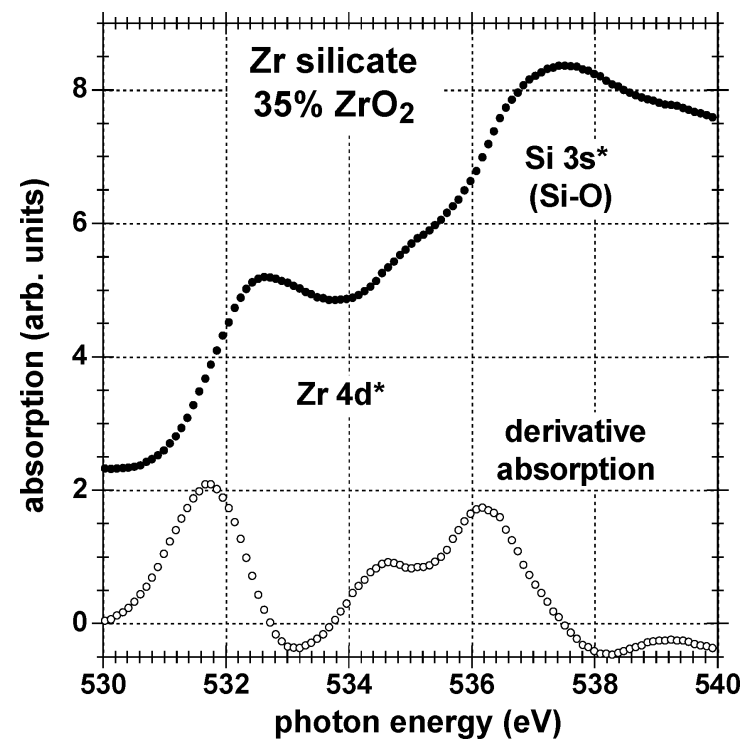

Fig. 7. $\mathrm{O} \mathrm{K}_{1}$ edge and differentiated $\mathrm{O} \mathrm{K}_{1}$ spectra for a noncrystalline $\mathrm{Zr}$ silicate alloy with $\sim 60$ molecular percent $\mathrm{ZrO}_{2}:\left(\mathrm{ZrO}_{2}\right)_{0.6}\left(\mathrm{SiO}_{2}\right)_{0.4}$. The dashed line markers indicate the positions of the spectral features in nanocrystalline $\mathrm{ZrO} 2$ thin films.

perovskite structure [4]. Stated differently the doubly degenerate $\mathrm{d}_{3 / 2}$ and triply degenerate $\mathrm{d}_{5 / 2}$ states are at the same energy giving rise to a d-state that is five fold degenerate. This degeneracy is removed in octahedral, tetragonal and cubic bonding environments as indicated in the diagram. The removal of this d-state degeneracy is generally designated as a crystal field splitting, and has been described at length in texts that have addressed TM/RE molecules and crystalline solids [3], [4], [15]. Other bonding environments with lower symmetry, such as the bonding of a TM atom at the center of a square also remove the degeneracies of the $d_{3 / 2}$ and $d_{5 / 2}$ states as well [15].

Crystal field splittings of the type described above are observed in noncrystalline $\mathrm{Zr}$ and $\mathrm{Hf}$ silicate alloys, and a representative $\mathrm{O} \mathrm{K}_{1}$ edge spectrum for a $\mathrm{Zr}$ silicate alloy ( $\left.\sim 35 \% \mathrm{ZrO}_{2}\right)$ is presented in Fig. 7. There is no evidence in the absorption spectrum, or the derivative of the absorption spectrum of this representative silicate alloy for any additional d-state multiplicity indicative of a further removal of d-state degeneracy. The $\mathrm{O} \mathrm{K}_{1}$ spectrum (not shown) of this $\mathrm{Zr}$ silicate alloy indicates are reduction of the 4d-state line-width after an anneal at $900{ }^{\circ} \mathrm{C}$ which results in chemical phase separation into crystalline $\mathrm{ZrO}_{2}$ and noncrystalline $\mathrm{SiO}_{2}$ [7].

In marked contrast are the spectra in Figs. 6 and 5(a) and (b). Fig. 6 indicates the $\mathrm{L}_{3}$ edge spectrum of $\mathrm{TiO}_{2}$ in which five features are clearly evident. The number of distinct features in consistent with the complete removal of the $d_{5 / 2}$ and $d_{3 / 2}$ degeneracies. The lower energy $\mathrm{T}_{2 \mathrm{~g}}$ triplet displays approximately equal splittings of $0.75 \pm 0.5 \mathrm{eV}$ between these three states, and the higher energy $\mathrm{E}_{\mathrm{g}}$ doublet has an approximate splitting of $0.8 \pm$ $0.2 \mathrm{eV}$. These term-splittings are consistent with the distorted octahedral bonding environment of $\mathrm{Ti}$ in crystalline $\mathrm{TiO}_{2}$ with a rutile structure [3], [4], [11]. The octahedral arrangement of $\mathrm{O}$ atoms about the $\mathrm{Ti}$ atom displays a symmetric Ti-O bonding elongation of about $0.003 \mathrm{~nm}$ in the $\mathrm{z}$-direction; the bonds in the $x$-y plane are the same length, but the square arrangement of an ideal octahedron in this plane is converted to a rectangle by an approximately seven degre $\left(7^{\circ}\right)$ bond angle change [11]. These degeneracy removals are commonly referred to as static Jahn-Teller term splittings, since the distortion increases the binding energy. This is contrast to dynamic Jahn-Teller splittings that occur during optical or near UV excitation [3], [15]. The increased bonding energy comes about because the number of d-electrons is two, and these occupy a lower energy state after the symmetric $T_{2 g}$ term splitting. These term splittings are not evident in the $\mathrm{TiO}_{2} \mathrm{O} \mathrm{K}_{1}$ edge spectrum of $\mathrm{TiO}_{2}$, nor in the differentiation of the that spectrum as well, but as will discussed later on in this paper are clearly evident in both the photoconductivity response and in the dielectric constant and absorption constant extracted from spectroscopic ellipsometry measurements.

It is important to note that in nonmolecular solids, these term splittings can only occur in a crystalline environment because they require symmetry reductions that involve second neighbors as well. The Jahn-Teller term splittings can also be viewed as a special class of crystal field effects; however, this nomenclature does not provide an insight into the forces that contribute to the distorted bonding environment; i.e., an increase in binding energy associated with occupancy of the lowest term split state. The evolution of symmetry reductions that result from distortions of octahedral, as well as cubic and tetrahedral bonding in the context of the Jahn-Teller effect are displayed in Fig. 1, and include the octahedral and cubic distortions important for: 1) Ti and Sc, and 2) $\mathrm{Zr}$ and $\mathrm{Hf}$, respectively.

In marked contrast to the results described above for $\mathrm{TiO}_{2}$, the $\mathrm{O} \mathrm{K}_{1}$ derivative spectra in Fig. 5(a) and (b) indicate a complete removal of d-state degeneracies in nanocrystalline $\mathrm{ZrO}_{2}$ and $\mathrm{HfO}_{2}$, respectively. These degeneracy removals are not evident in the $\mathrm{Zr} \mathrm{M}$ and $\mathrm{Hf} \mathrm{N}_{3}$ spectra, consistent with a significantly longer core hole life-time associated with the $\mathrm{O} 1 \mathrm{~s}$ state than for the $\mathrm{Zr} 3 \mathrm{p}$ and $\mathrm{Hf} 4 \mathrm{p}$ states. Core hole life-times scale inversely as the atomic number, $\mathrm{Z}^{\mathrm{n}}$, of the atom from which the excitation occurs, where $n \sim 2-3$.

Returning to the differentiated spectra in Fig. 5(a) and (b), the lower energy components of the $\mathrm{d}^{*}$-state spectral features are split into two states and the higher frequency components into three states. This is consistent with the Jahn-Teller term splittings in cubic bonding environments in which there are distortions along all three principal axes. For distorted cubic bonding, the doublet $\mathrm{E}_{\mathrm{g}}$ or $\mathrm{d}_{3 / 2}$ state is lower in energy than the triplet $\mathrm{T}_{2 \mathrm{~g}}$ or $\mathrm{d}_{5 / 2}$ state, or exactly the reverse of the ordering in an octahedral environment with distortions along the three coordinate axes. The d-state splittings for $\mathrm{HfO}_{2}$ are approximately 1.2 to $1.3 \mathrm{eV}$ and larger by about $50 \%$ compared to those for $\mathrm{TiO}_{2}$, providing a partial explanation for their observabilities in the $\mathrm{O} \mathrm{K}_{1}$ edge spectra.

Fig. 8 indicates the Jahn-Teller term splittings for $\mathrm{ZrO}_{2}$ and $\mathrm{HfO}_{2}$ spectral features in the $\mathrm{O} \mathrm{K}_{1}$ edge. This figure also includes the $\mathrm{TiO}_{2}$ spectral features. For $\mathrm{TiO}_{2}$, the highest energy state of the $\mathrm{T}_{2 \mathrm{~g}}$ triplet in the $\mathrm{Ti}_{3}$ spectrum has been set equal to the lower energy $3 \mathrm{~d}^{*}$ feature in the $\mathrm{O} \mathrm{K}_{1}$ spectrum. This approach is consistent with the spectra of $\mathrm{Zr}$ titanate alloys to be discussed in the next section of this paper. Finally, the average $d^{*}$-state energies obtained from this analysis of the respective 


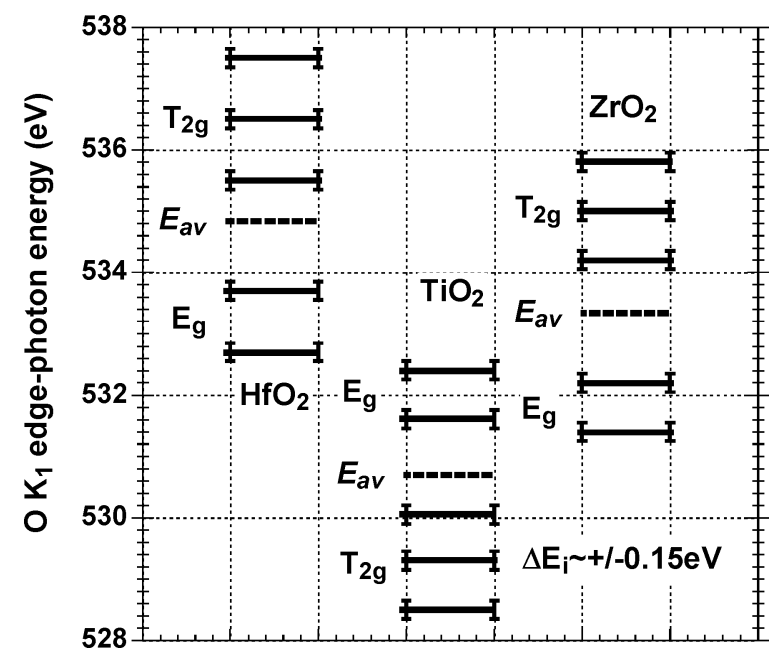

Fig. 8. Jahn-Teller term splittings for the $\mathrm{ZrO}_{2}$ and $\mathrm{HfO}_{2}$ spectral features in the $\mathrm{O} \mathrm{K}_{1}$ edge. This figure also includes the $\mathrm{TiO}_{2}$ spectral features that have been scaled from the $\mathrm{L}_{3}$ edge spectrum by equating the energies of the two strongest features in the $\mathrm{TiO}_{2} \mathrm{O} \mathrm{K}_{1}$ and $\mathrm{L}_{3}$ spectra. The diagram also includes the average energies, $E_{a v}$, of the respective d-state features.

$\mathrm{O} \mathrm{K}_{1}$ spectra scale monotonically with the atomic d-state energies of the atomic species, $\mathrm{Ti}, \mathrm{Zr}$, and $\mathrm{Hf}$.

\section{Band Gap and Conduction Band Offset Energy Scaling in} Elemental Oxides: The Motivation for Studying Band Edge States in Complex Oxides

Robertson has developed a charge neutrality level (CNL) model for determination of conduction band offset energies for high- $\kappa$ dielectrics formed on Si [2]. The results presented in [2] are plotted in this paper in Fig. 9 to reveal an approximately linear relationship for $E_{B}$ as a function of $E_{\text {gap }}$. Fig. 9(a) and (b) contains plots, respectively, of: 1) $\mathrm{E}_{\mathrm{B}}$ as a function of $E_{\text {gap }}$, and 2) $E_{\text {gap }}$ and $E_{B}$ as a functions of atomic d state energy for a representative set of TM oxides. The scaling in Fig. 9(b) follows directly from the spectroscopic results presented above, and from the ab initio calculations for $\mathrm{O} \mathrm{K}_{1}$ spectra that provide the correlation between optical bandgaps and atomic d-state energies [11]. A band offset energy of at least $1 \mathrm{eV}$, and more generally about $1.2 \mathrm{eV}$ or more is required for tunneling/thermionic leakage current to be sufficiently low for scaled CMOS devices [17]. Using this criterion, the TM oxides with the highest dielectric constants, $\mathrm{TiO}_{2}$, and $\mathrm{Nb}_{2} \mathrm{O}_{3}$ and $\mathrm{Ta}_{2} \mathrm{O}_{3}$, have offset energies below $1 \mathrm{eV}$ that either correlate with high tunneling leakage, and/or electric field assisted injection into low-lying conduction band-states associated with these atoms, and are therefore not candidates for replacement dielectrics in scaled CMOS devices. Based on this scaling with atomic d-states, the elemental oxides of: 1) $\mathrm{Zr}$ and $\mathrm{Hf}$ and 2) $\mathrm{Y}, \mathrm{La}$ and the lanthanide RE oxides are expected to have conduction band offset energies $>1.2 \mathrm{eV}$, and therefore have the potential for meeting roadmap targets for low tunneling leakage current, provided that the electron tunneling mass, $\mathrm{m}_{\mathrm{eff}}$, does not decrease significantly with decreasing $\mathrm{E}_{\mathrm{B}}$, and mitigate gains for increases in $\kappa$ [see (1)]. Since the TM and RE d-states do not mix with the lowest conduction band s-states of $\mathrm{Si}$ and $\mathrm{Al}$, these scaling arguments can be extended to the silicate

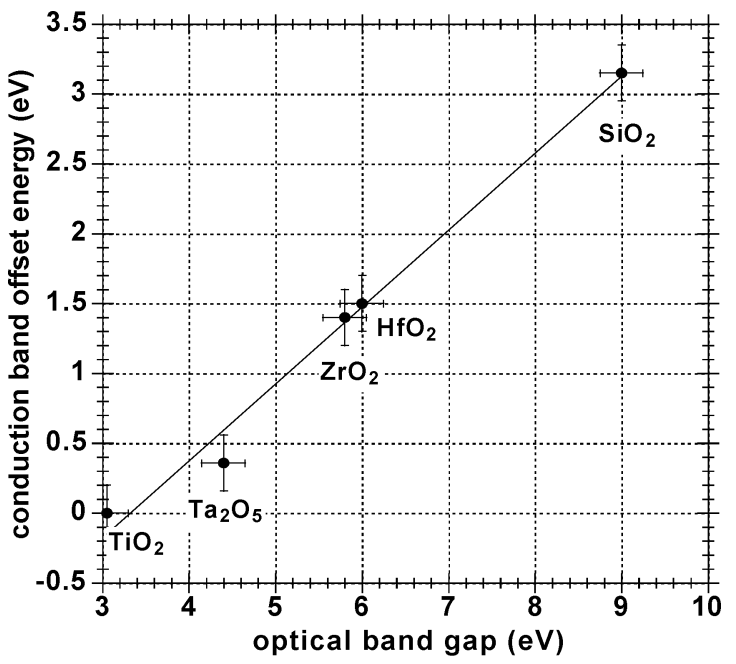

(a)

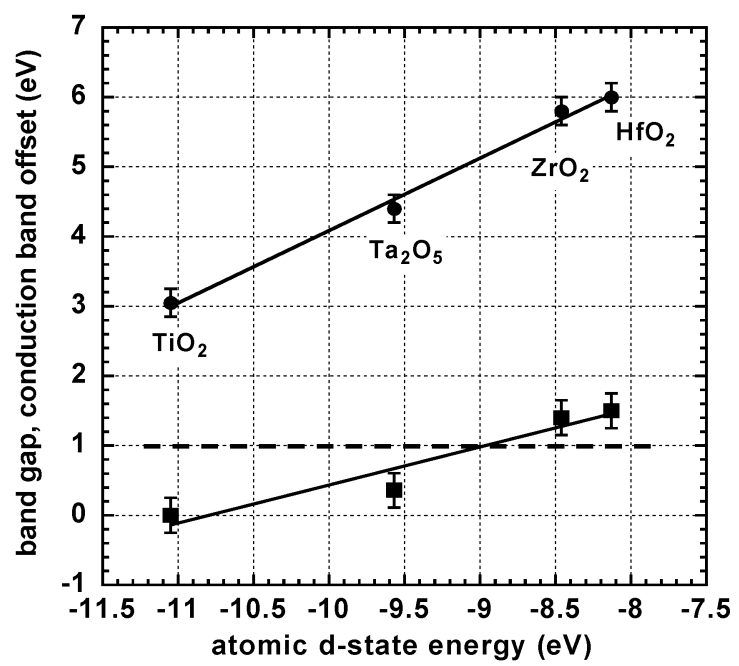

(b)

Fig. 9. (a) Conduction band offset energy, $E_{B}$, as a function of the optical bandgap, $E_{g}$, from the results presented in [2]. (b) $E_{g}$ and $E_{B}$ as a function of atomic d-state energy.

and aluminate alloys of the $\mathrm{Zr}, \mathrm{Hf}, \mathrm{Y}, \mathrm{La}$ and the lanthanide $\mathrm{RE}$ atoms [7]. However, it must be recognized that there are many other factors that contribute to the integration of high- $\kappa$ dielectrics into advanced Si devices [1]. These include process integration issues relative to thermal budgets, substitution of dual metal or single midgap metal gates for bulk and thin film Si on insulator devices, respectively, as well as other high- $\kappa$ dielectric issues including: 1) internal dielectric interfaces with ultrathin interfacial $\mathrm{SiO}_{2}$ layers [18], [19], and 2) intrinsic band edge traps addressed in this paper [20], [21].

The results presented above have raised an interesting question regarding the lowest energy $\mathrm{d}^{*}$-states in complex oxides that include $\mathrm{Sc}$ and $\mathrm{Ti}$, as for example stoichiometric and $\mathrm{Zr}$ and Hf titanates, and RE scandates such as La, Dy and Gd scandate: is it possible for d-states of the two TM atoms in the titanates, and the Sc and RE atoms of the scandates to couple and mix in ways that increase the optical bandgap, and the conduction band offset energies of these complex alloys? If this mixing were to occur this would then provide a way to obtain independent control of band offset energies and dielectric constants, and thereby 


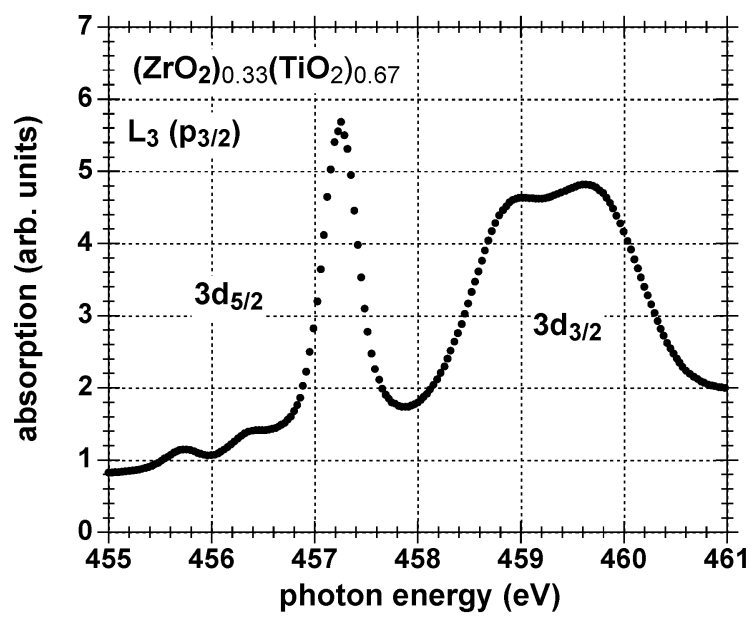

(a)

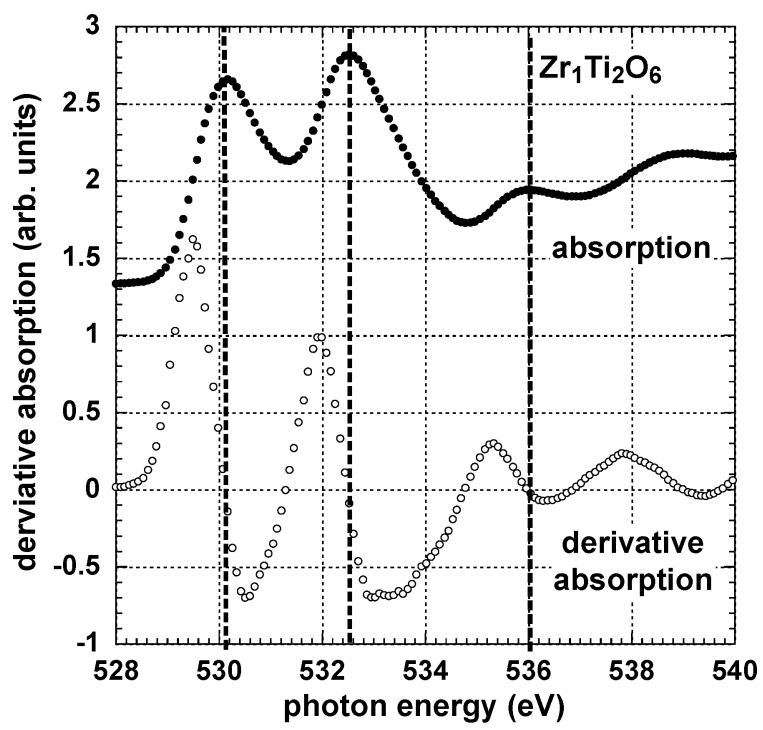

(b)

Fig. 10. Core level $\mathrm{x}$-ray absorption spectra for stoichiometric $\mathrm{Zr}$ titanate alloy with a composition, $\mathrm{ZrTi}_{2} \mathrm{O}_{6}$ or $\left(\mathrm{ZrO}_{2}\right)_{0.33}\left(\mathrm{TiO}_{2}\right)_{0.67}$ : (a) the $\mathrm{Ti} \mathrm{L}_{2,3}$ spectrum and (b) the $\mathrm{O} \mathrm{K}_{1}$ spectrum. The dashed lines in indicate the $\mathrm{d}^{*}$-state features in the $\mathrm{O} \mathrm{K}_{1}$ spectrum in (b).

get around the limitations of Fig. 9(a) and (b). These issues are addressed in Section IV.

\section{COMPLEX OXIDES}

There has been an increasing interest in stoichiometric complex oxides as high- $\kappa$ dielectrics for scaled Si CMOS. These oxides are differentiated from TM/RE silicate and aluminates which are pseudo-binary alloys, e.g., $\left(\mathrm{ZrO}_{2}\right)_{\mathrm{x}}\left(\mathrm{SiO}_{2}\right)_{1-\mathrm{x}}$, in which the alloy compositions are varied continuously with the range, $1.0>\mathrm{x}>0.0$. Two different alloy systems are addressed; these are: 1) $\mathrm{Zr}$ and $\mathrm{Hf}$ titanates, in which the values $\mathrm{x}$ for $\mathrm{ZrO}_{2}$ and $\mathrm{HfO}_{2}$ to $1-\mathrm{x}$ for $\mathrm{TiO}_{2}$ have been restricted to compositional ratios of $1: 2,1: 1$ and $2: 1$, and 2) the stoichiometric RE scandates, e.g., $\mathrm{La}(\mathrm{Dy}, \mathrm{Gd}) \mathrm{ScO}_{3}$ in which the ratio of the elmental oxides, $\mathrm{La}(\mathrm{Dy}, \mathrm{Gd})_{2} \mathrm{O}_{3}$ and $\mathrm{Sc}_{2} \mathrm{O}_{3}$ are 1:1.

Analysis of XAS spectra for these materials have demonstrates that there is very little coupling between the d-states of

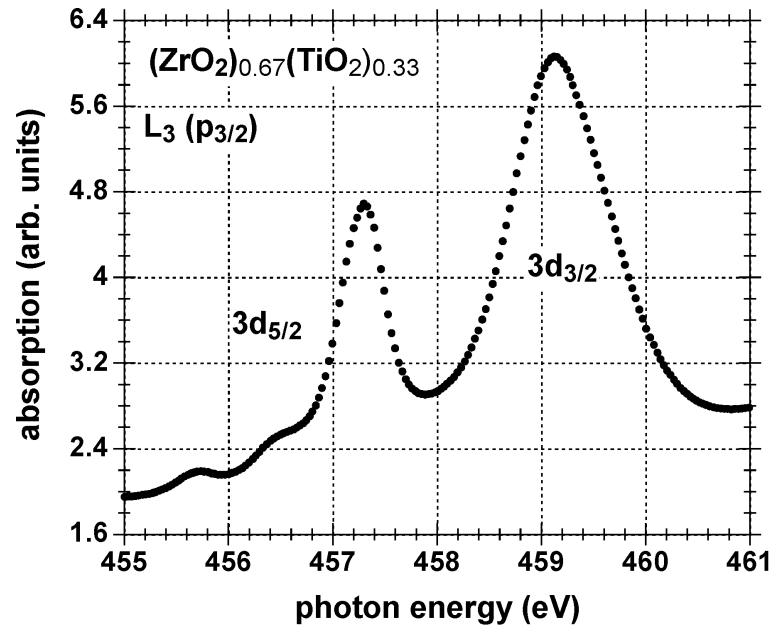

(a)

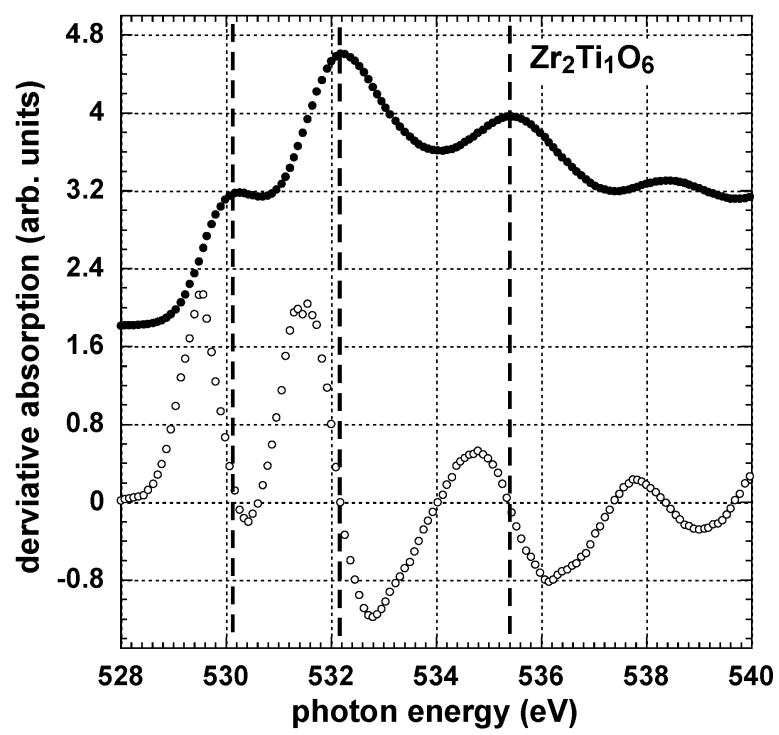

(b)

Fig. 11. Core level x-ray absorption spectra for stoichiometric Zr titanate alloy with a composition, $\mathrm{Zr}_{2} \mathrm{TiO}_{6}$ or $\left(\mathrm{ZrO}_{2}\right)_{0.67}\left(\mathrm{TiO}_{2}\right)_{0.33}$ : (a) the $\mathrm{Ti}_{2,3}$ spectrum and (b) the $\mathrm{O} \mathrm{K}_{1}$ spectrum. The dashed lines in indicate the $\mathrm{d}^{*}$-state features in the $\mathrm{O} \mathrm{K}_{1}$ spectrum in (b).

the constituent TM and/or RE d-states. To a very good approximation, the d-state features in the respective $\mathrm{O} \mathrm{K}_{1}$ edges are at energies which are closely coupled to their atomic d-state energies in the $\mathrm{nd}^{2}(\mathrm{n}+1) \mathrm{s}^{2}$ representation for tetravalent group IVB, and in the $\mathrm{nd}^{1}(\mathrm{n}+1) \mathrm{s}^{2}$ representation for trivalent TM and lanthanide series RE metals discussed above. This aspect of the spectra will be addressed with respect to the $\mathrm{ZrTi}_{2} \mathrm{O}_{6}, \mathrm{Zr}_{2} \mathrm{TiO}_{6}$, and $\mathrm{LaScO}_{3}$ thin films discussed in Sections IV-A and IV-B.

\section{A. $\mathrm{Zr}$ and $\mathrm{Hf}$ Titanates}

Fig. 10(a) displays the $\mathrm{L}_{3}$ Ti spectrum, and Fig. 10(b) the $\mathrm{O} \mathrm{K}_{1}$ spectrum for a stoichiometric alloy with a composition, $\mathrm{ZrTi}_{2} \mathrm{O}_{6}$. Fig. 11(a) and (b) displays the corresponding spectra for a second alloy with a composition $\mathrm{Zr}_{2} \mathrm{TiO}_{6}$. The $\mathrm{O} \mathrm{K}_{1}$ edge spectra in Figs. 10(b) and 11(b), respectively, indicate three distinct $\mathrm{d}^{*}$-state spectral features, the energies of which are consistent with a mixture of features terminating in transitions to the respective $\mathrm{Ti}$ and $\mathrm{Zr}$ d-state at approximately: 1) 530.2 and 
$532.4 \mathrm{eV}$ for $\mathrm{TiO}_{2}$ and 2) 532.4 and 535.4 to $536.0 \mathrm{eV}$ for $\mathrm{ZrO}_{2}$, each with an experimental uncertainty of $\pm 0.1 \mathrm{eV}$. This means that the lowest energy spectral feature is $\mathrm{TiO}_{2}$-like, the highest spectral feature $\mathrm{ZrO}_{2}$-like, and the middle frequency has contributions from both $\mathrm{TiO}_{2}$ and $\mathrm{ZrO}_{2}$. Derivative $\mathrm{O} \mathrm{K}_{1}$ spectra do not indicate and additional $\mathrm{d}^{*}$-state spectral features. The respective $\mathrm{Ti} \mathrm{L}_{3}$ spectra in Figs. 10(a) and 11(a), provide evidence for Jahn-Teller term splittings indicating a complete removal of the Ti $3 \mathrm{~d}$ state degeneracies. The presence of the term split features in the $\mathrm{Ti}_{3}$ spectra, and their absence in the respective $\mathrm{O} \mathrm{K}_{1}$ edge spectra is essentially the same behavior noted above for $\mathrm{TiO}_{2} \mathrm{O} \mathrm{K}_{1}$ and $\mathrm{Ti}_{3}$ spectra. The $\mathrm{T}_{2 \mathrm{~g}}$ term-split energies of the three lower $\pi$-states in the $\mathrm{Ti} \mathrm{L}_{3}$ spectra are the same for each composition, whereas there are marked differences in the two $\mathrm{E}_{\mathrm{g}}$ term-splitting of the $\sigma$-states. These are consistent with differences in the character of the bonding between $\mathrm{Ti}$ and its second neighbors: 1) a mixture of ionic and covalent bonding for a compositionally averaged mixture of $\mathrm{Zr}$ and Ti neighbors for $\mathrm{ZrTi}_{2} \mathrm{O}_{6}$, and 2) a more ionic bonding with a compositional average two $\mathrm{Zr}$ neighbors in $\mathrm{Zr}_{2} \mathrm{TiO}_{6}$ [3]. The second neighbor bonding has little effect on the $\mathrm{T}_{2 \mathrm{~g}} \pi$-bonding states which are localized on the nearest neighbor Ti and $\mathrm{O}$, but has a strong influence on the $\mathrm{E}_{\mathrm{g}} \sigma$-bonding states which are significantly more delocalized.

The $\mathrm{O} \mathrm{K}_{1}$ spectra of $\mathrm{Hf}$ titanate alloys, $\left(\mathrm{HfO}_{2}\right)_{\mathrm{m}}\left(\mathrm{SiO}_{2}\right)_{\mathrm{n}}$, with: 1) $\mathrm{m}=1, \mathrm{n}=2, \mathrm{~m}=1, \mathrm{n}=2$, and $\mathrm{m}=2$ and $\mathrm{n}=1$ display qualitatively and quantitative similar spectra to the $\mathrm{Zr}$ titanates discussed above. To a good approximation, the features in the $\mathrm{O} \mathrm{K}_{1}$ edge are also well described by a mixture of $\mathrm{HfO}_{2}$ and $\mathrm{TiO}_{2}$ features, with small shifts that are consistent with a weak coupling between $\mathrm{Ti}$ and $\mathrm{Hf}$ d-states with the same symmetries. This is illustrated in Fig. 12(a) and (b) for $\mathrm{HfTiO}_{4}$, or the 1:1 composition: $\left(\mathrm{HfO}_{2}\right)_{1}\left(\mathrm{SiO}_{2}\right)_{1}$. The TM $\pi$-states that form the top of the valence band and the bottom of conduction band are predominantly Ti-like and are shifted by less than $0.5 \mathrm{eV}$ from their values in the $\mathrm{TiO}_{2}$, whereas there are larger shifts, $\sim 1 \mathrm{eV}$ in the Hf-like $\sigma$-bond states that are at the bottom of the valence band higher in the conduction band. One explanation proposed for this behavior was that it was indicative of relatively weak coupling between the $\pi$-bonded states that are at the top of valence band, and stronger coupling between the $\sigma$-bonded states that are deeper in the valence band [8]. However, this explanation fails to take into account the strong contribution of $\mathrm{O} 2 \mathrm{p}$ nonbonding states at the top of the valence band [6]. On the other hand, the differences in the valence band states of the elemental and complex oxides are explained quantitatively by noting that the valence and conduction band spectra of the constituent elemental oxides, $\mathrm{TiO}_{2}$ and $\mathrm{HfO}_{2}$ include the effects of Jahn-Teller term splittings, whereas those of the complex oxide, $\mathrm{HfTiO}_{4}$ include Jahn-Teller shifts for the Ti-atoms, but not the Hf-atoms. The $\sim 1 \mathrm{eV}$ shifts of the states at the bottom of the valence band, and above the conduction band edge as revealed in the $\mathrm{O} \mathrm{K}_{1}$ spectrum. The smaller shifts in the states at the top of valence band and bottom of the conduction band may indeed be due to a weak coupling of $\pi$-bonded states; however, this will require additional study.

Similar valence band, and $\mathrm{O} \mathrm{K}_{1}$ edge shifts have been found in $\mathrm{Zr}$ titanate alloys, and additional compositions are being

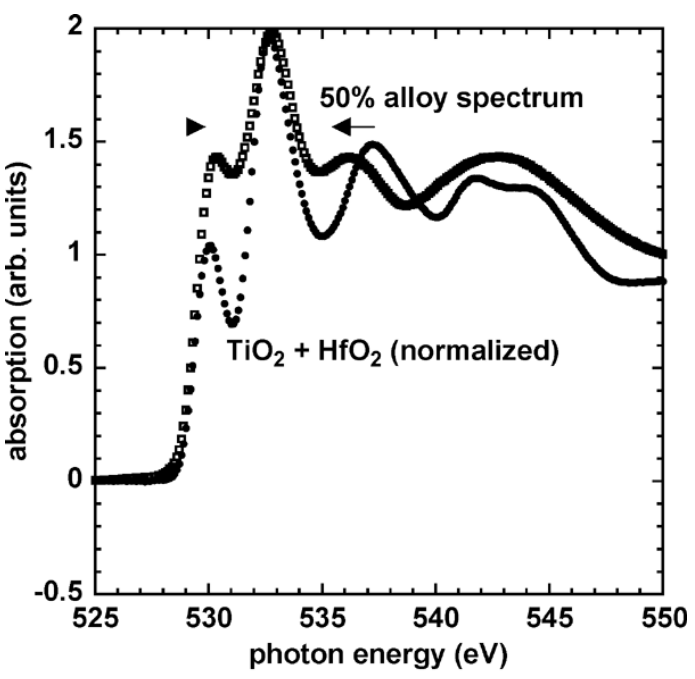

(a)

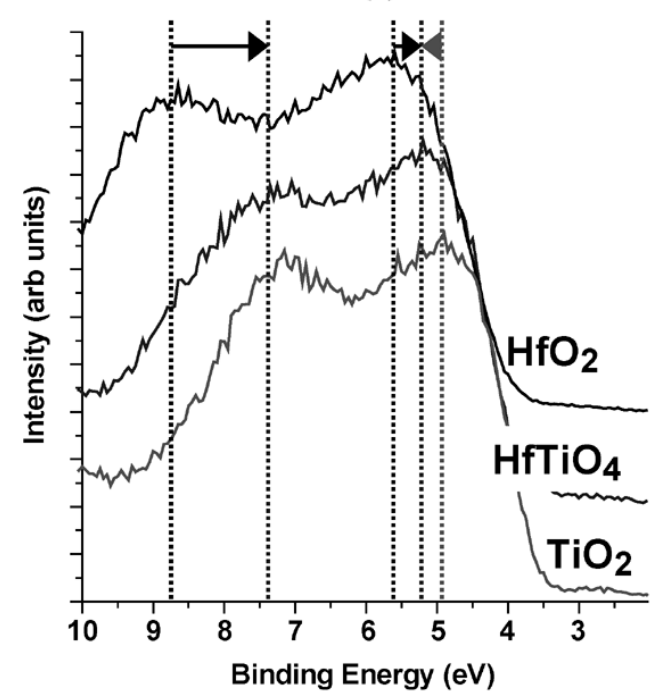

(b)

Fig. 12. (a) $\mathrm{O} \mathrm{K}_{1}$ and (b) UPS valence band spectra for $\mathrm{HfTiO}_{4}$, or equivalently an $\mathrm{Hf}$ titante alloy with $1: 1$ composition of $\mathrm{HfO}_{2}$ and $\mathrm{TiO}_{2}$. The arrows in (a) and (b) indicate the smaller shifts in the $\pi$-bonded states at the top of the valence band and bottom of the conduction, as well as the larger shifts in the $\sigma$-bonded states at the bottom of the valence band and at higher energies in the conduction band.

studied to see if the observed shifts correlate with $\mathrm{Zr}$ titanate phases, or mixtures of $\mathrm{ZrO}_{2}$ and $\mathrm{HfO}_{2}$. Stated otherwise, comparisons between $\mathrm{Zr}$ titanate alloys and the end-member oxides may prove to be a novel way of identifying compositions that correspond to stoichiometric complex oxides, as distinguished from alloys with random, or statistical bonding of the constituent elemental oxides.

\section{B. La and Other RE Scandates}

Qualitatively similar behavior are presented for stoichiometric $\mathrm{La}$, Dy, and Gd scandates. $\mathrm{Sc} \mathrm{L}_{2,3}, \mathrm{La} \mathrm{M}_{2,3}$ and $\mathrm{O} \mathrm{K}_{1}$ spectra are presented in Fig. 13(a), (b), and (c), respectively, for $\mathrm{LaScO}_{3}$ or equivalently $\left(\mathrm{La}_{2} \mathrm{O}_{3}\right)_{1}\left(\mathrm{Sc}_{2} \mathrm{O}_{3}\right)_{1}$ that has been annealed at $1000{ }^{\circ} \mathrm{C}$ in an inert ambient. The $\mathrm{Sc} \mathrm{L}_{2,3}$ spectrum in Fig. 13(a) displays a Jahn-Teller term-splitting similar to those of the Ti spectrum for $\mathrm{Zr}_{2} \mathrm{TiO}_{6}$ in Fig. 11(a), where the 


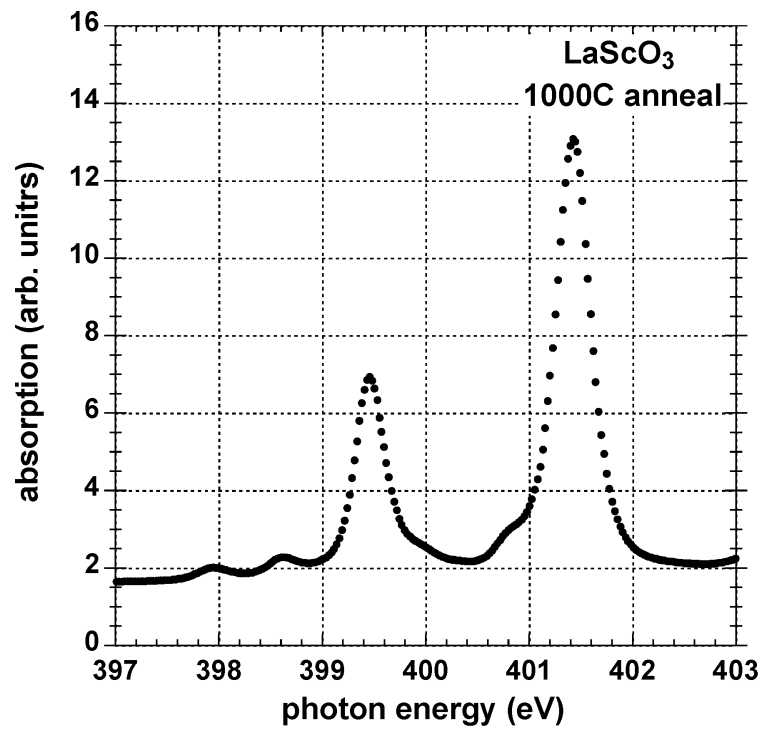

(a)

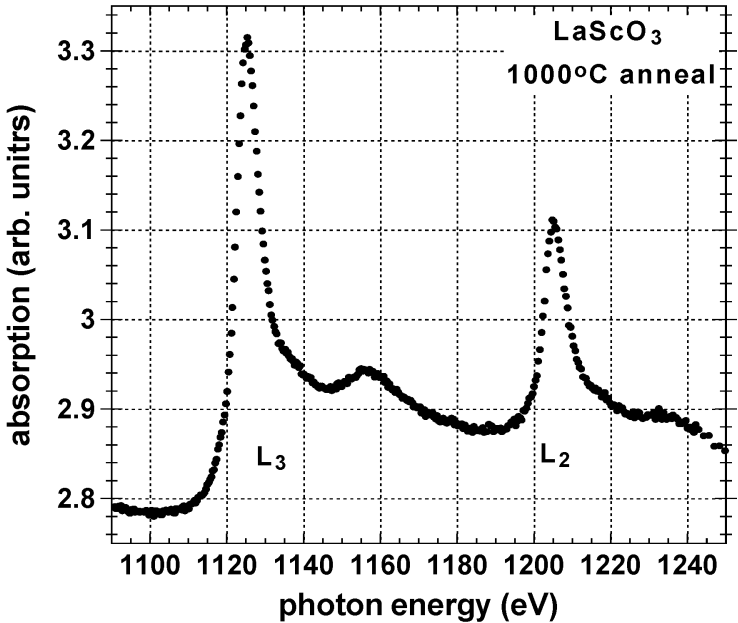

(b)

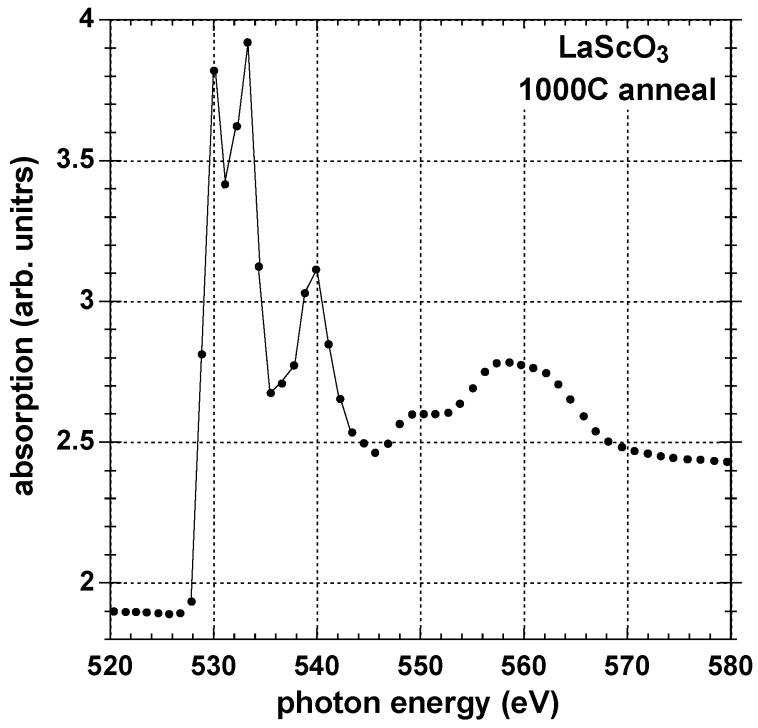

(c)

Fig. 13. Core level XAS spectra for nanocrystalline $\mathrm{LaScO}_{3}$ or equivalently $\left(\mathrm{La}_{2} \mathrm{O}_{3}\right)_{0.5}\left(\mathrm{Sc}_{2} \mathrm{O}_{3}\right)_{0.5}$ that has been annealed at $1000{ }^{\circ} \mathrm{C}$ in an inert ambient: (a) $\mathrm{Sc} \mathrm{L}_{2,3}$ spectrum, (b) $\mathrm{La} \mathrm{M}_{2,3}$ spectrum, and (c) $\mathrm{O} \mathrm{K}_{1}$ spectrum.

term-splitting of the $\mathrm{E}_{\mathrm{g}}$ feature is indicative of ionic bonding. The $\mathrm{T}_{\mathrm{g}}$ term-splittings are $0.80 \pm .05 \mathrm{eV}$, and are approximately the same as in $\mathrm{TiO}_{2}$ and the $\mathrm{Zr}$ and $\mathrm{Hf}$ titanate alloys; however the average $\mathrm{T}_{2 \mathrm{~g}}-\mathrm{E}_{\mathrm{g}}$ or crystal field splitting is less, $\sim 2.7 \mathrm{eV}$ as compared to $3.1 \mathrm{eV}$.

The $\mathrm{La} \mathrm{M}_{2,3}$ spectrum is displayed in Fig. 13(b). There is only one $5 \mathrm{~d}$ feature in each of the spin-orbit split spectra. This is confirmed by taking a derivative spectrum, and also by comparison with the $\mathrm{La} \mathrm{M}_{2,3}$ spectrum in $\mathrm{LaAlO}_{3}$, where each of the spin orbit spectra shows multiple La $5 \mathrm{~d}$ states. The combination of spectroscopically resolved Jahn-Teller splittings in the $\mathrm{Sc}_{2,3}$ spectrum in Fig. 13(a) and the absence of Jahn-Teller splittings in the $\mathrm{La} \mathrm{M}_{2,3}$ spectrum in Fig. 13(b) is consistent with a nanocrystalline morphology based on the perovskite structure. In this structural model, there are $\mathrm{x}, \mathrm{y}$ and $\mathrm{z}$ displacements of the Ti atom respect to its six O-neighbors, but the $\mathrm{La}$ atom remains in a symmetric environment with twelve nearest-neighbor
$\mathrm{O}$-atoms. The crystalline nature of this film has been verified by $\mathrm{x}$-ray diffraction (XRD) [22].

The interpretation of the $\mathrm{Sc} \mathrm{L}_{2,3}$ and $\mathrm{La} \mathrm{M}_{2,3}$ spectra are consistent with the multiplicity of features in the $\mathrm{O} \mathrm{K}_{1}$ edge in Fig. 13(c). The spectra obtained to date are survey spectra at a lower spectral resolution than those obtained previously for crystalline $\mathrm{GdScO}_{3}$ and $\mathrm{DyScO}_{3}$ [9], and thin film $\mathrm{DyScO}_{3}$ (not shown); however the $\mathrm{O} \mathrm{K}_{1}$ spectrum for the $1000{ }^{\circ} \mathrm{C}$ annealed film is consistent with the assignments made for $\mathrm{Gd}$ and Dy scandate $\mathrm{O} \mathrm{K}_{1}$ spectra in [2]. The feature at $\sim 538$ to $540 \mathrm{eV}$ in the $\mathrm{LaScO}_{3} \mathrm{O} \mathrm{K}_{1}$ spectrum is interpreted as the La $5 \mathrm{~d}$ feature with a five-fold degeneracy similar to what has been found in the $\mathrm{La} \mathrm{M}_{2,3}$ spectrum. The lower energy features at $\sim 530 \mathrm{eV}$ and $532 \mathrm{eV}$ are corresponding to the two strongest features in the $\mathrm{Sc} \mathrm{L}_{2,3}$ spectrum at $\sim 399.4$ and $401.4 \mathrm{eV}$. The splitting between these features in the Sc $L_{2,3}$ spectrum is $\sim 2.0 \pm 0.1 \mathrm{eV}$, and approximately 2.2 


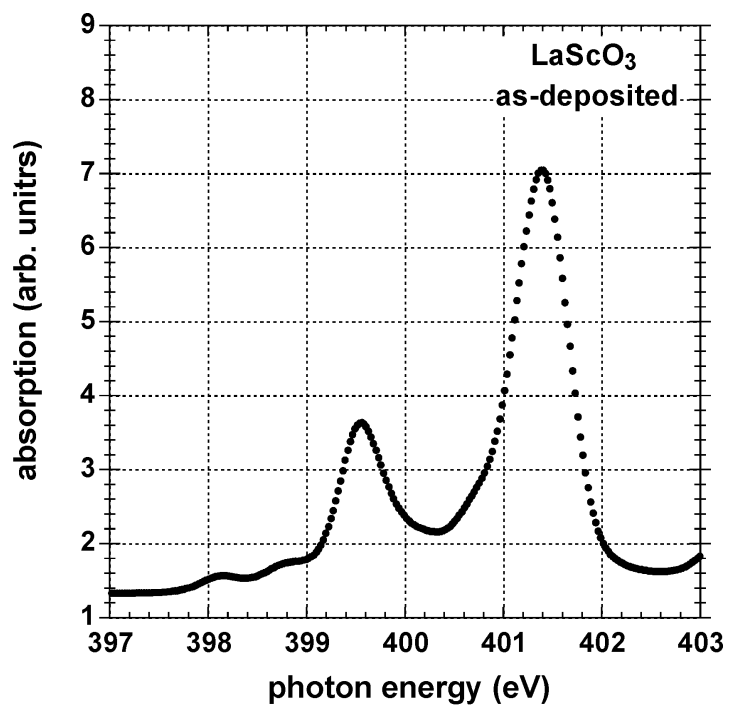

(a)

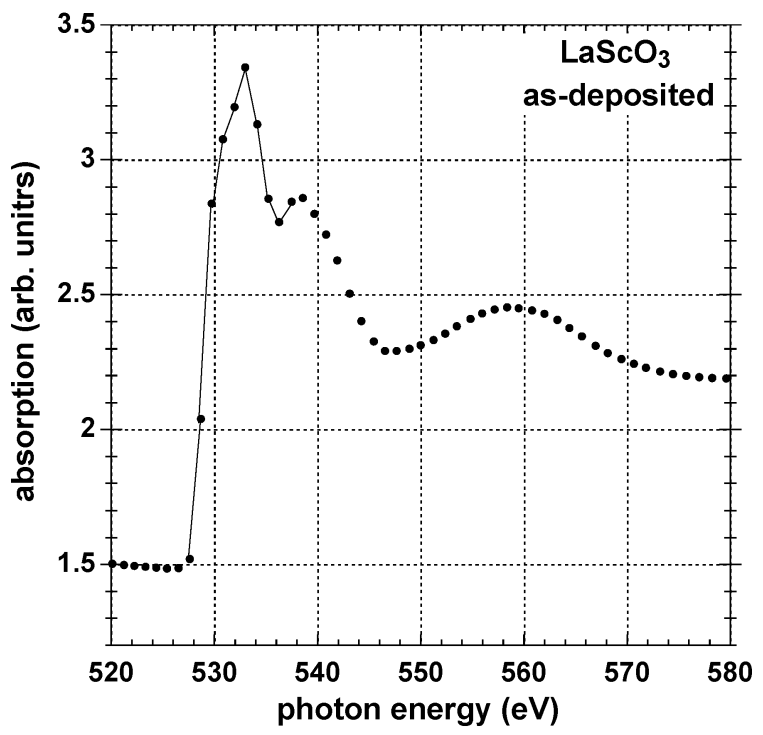

(b)

Fig. 14. Core level XAS spectra for as-deposited nanocrystalline $\mathrm{LaScO}_{3}$ : (a) $\mathrm{Sc}_{2,3}$ spectrum, and (b) $\mathrm{O} \mathrm{K}_{1}$ spectrum.

$\pm 0.25 \mathrm{eV}$ in the $\mathrm{O} \mathrm{K}_{1}$ edge. This interpretation is consistent with the interpretation of the $\mathrm{Zr}$ titanate spectra discussed above; i.e., the $\mathrm{TiO}_{2}$-like features in the $\mathrm{O} \mathrm{K}_{1}$ edge have a separation that is essentially the same as the separation between the two strongest features in the $\mathrm{Ti}^{\mathrm{L}} \mathrm{L}_{2,3}$ spectrum.

Fig. 14(a) and (b) shows the $\mathrm{Sc}_{2,3}$ and the $\mathrm{O} \mathrm{K}_{1}$ edge spectra for as-deposited films, while Fig. 15(a) and (b) shows the same spectra for films annealed at $700{ }^{\circ} \mathrm{C}$. The term-splittings in the Figs. 14(a) and 15(a), as well as the absence of term, or crystal field splitting the correspond as-deposited and $700{ }^{\circ} \mathrm{C}$ annealed La $\mathrm{M}_{2,3}$ spectra (not shown) confirm the nanocrystalline nature of these films. XRD studies have detected crystallinity after an $850{ }^{\circ} \mathrm{C}$ anneal, but not in as-deposited or $700{ }^{\circ} \mathrm{C}$ annealed samples. This establishes that the XAS spectra are more sensitive to nanocrystallinity than XRD [22]; e.g., XRD can detect nanocrystallinity at a scale greater than $5 \mathrm{~nm}$, but not at scale of less than $3 \mathrm{~nm}$.

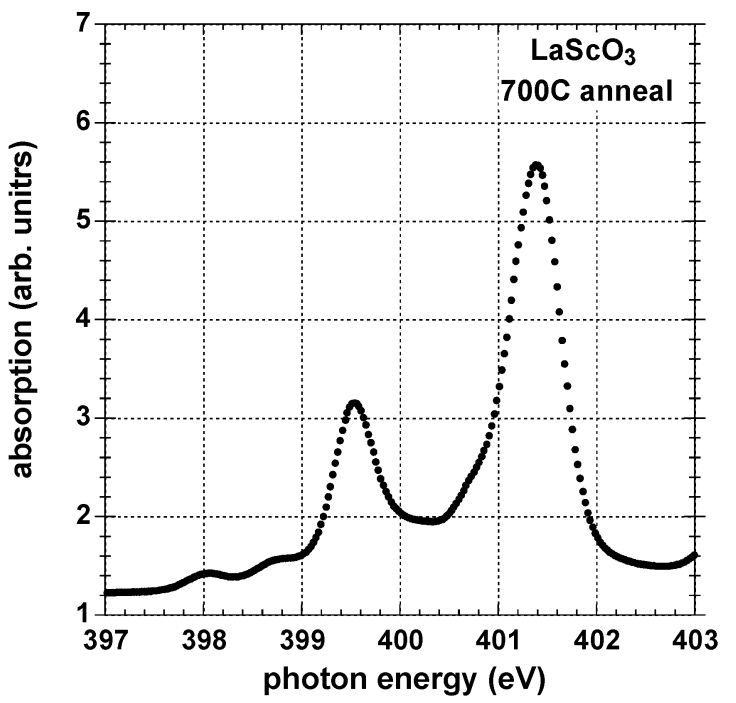

(a)

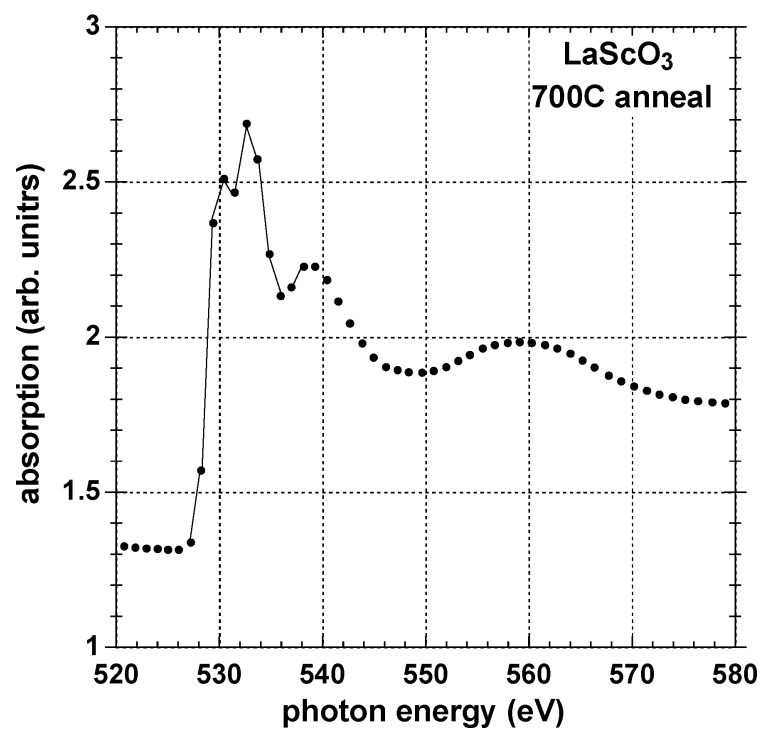

(b)

Fig. 15. Core level XAS spectra for nanocrystalline $\mathrm{LaScO}_{3}$ that has been annealed at $700{ }^{\circ} \mathrm{C}$ in an inert ambient: (a) $\mathrm{Sc} \mathrm{L}_{2,3}$ spectrum, and (b) $\mathrm{O} \mathrm{K}_{1}$ spectrum.

\section{DISCUSSION}

\section{A. Spin Orbit, Crystal Field, and Jahn-Teller Term Splittings}

There are quantitative differences between: 1) spin-orbit splittings in core and valence d-states, and 2) d-state splittings that result from the electrostatic fields of near-nearest and more distant neighbors. Spin-orbit splittings dominate in core states, with the splitting increasing as the atomic number of the atom increases and the principal quantum number decreases. In contrast spin orbit splittings in valence $\mathrm{p}$ - and d-states are typically much less than $1 \mathrm{eV}$, and are lower by factors of at least two to crystal field spittings. Fig. 1 indicates a schematic representation of the crystal field and Jahn-Teller reductions in atomic d-state degeneracies that result from nearest neighbor bonding, and reductions/distortions in nearest-neighbor bonding and beyond that result from the static Jahn-Teller effect [2], [3], [15]. A cluster model to be published elsewhere, based on the Ph.D. 
dissertation of one of the co-authors [11], has been proven useful in tracking some of the changes in d-state character in Fig. 6.

For example, when applied to $\mathrm{ZrO}_{2}$, a cluster based on tetrahedral coordination give a smaller $4 \mathrm{~d}$ crystal field splitting than a cubic eight-fold coordination cluster, $\sim 1.9 \mathrm{eV}$ as compared to $2.7 \mathrm{eV}$, scaling approximately as the square root of the number of nearest neighbor $\mathrm{O}$ atoms. Additionally, when applied to $\mathrm{TiO}_{2}$, a cluster based on a distorted octahedral bonding arrangement that replicates the local atomic structure in rutile, removed the respective $T_{2 g}$ and $E_{g}$ symmetries, and gave Jahn-Teller term splitting in good agreement with the $\mathrm{Ti}_{3}$ spectra of Figs. 3(a), 6, 10(a) and 11(a).

The cluster calculations do not consider spin orbit coupling, and since they gave very good agreement with experiment, this supports the argument that crystal field and Jahn-Teller term splittings dominate in valence band and conduction d-state energies in TM metal oxides, i.e., the crystal field and Jahn-Teller term splittings are greater than $0.7 \mathrm{eV}$ in Ti and $\mathrm{Sc} 3 \mathrm{~d}$-states, $\mathrm{Zr}$ $4 \mathrm{~d}$ and for Hf $5 \mathrm{~d}$ states.

\section{B. XAS Spectra of Elemental Oxides and Silicate Alloys}

Differentiated $\mathrm{O} \mathrm{K}_{1}$ spectra for films of $\mathrm{ZrO}_{2}$ and $\mathrm{HfO}_{2}$ prepared by remote plasma assisted deposition and annealed at temperatures sufficient to drive out any water or carbon impurities indicate a multiplicity of d-state features. The respective multiplicities indicate that the $\mathrm{E}_{\mathrm{g}}$ states are at the bottom of the conduction band, and therefore at the top of the valence band, and the $T_{2 g}$ states are higher in the conduction band, and deeper in the valence band [8]. The effective width of the valence band for $\mathrm{ZrO}_{2}$ is approximately $6 \mathrm{eV}$ for $\mathrm{ZrO}_{2}$, and approximately the same for $\mathrm{HfO}_{2}$ as well. The energy difference between the corresponding anti-bonding states in the respective $\mathrm{O} \mathrm{K}_{1}$ edges of $\mathrm{ZrO}_{2}$ and $\mathrm{HfO}_{2}$ are approximately the same and about $5 \mathrm{eV}$. The effective valence and conduction band widths are in excellent agreement with the calculations of [23].

The Jahn-Teller term-splittings in the $\mathrm{ZrO}_{2}$ and $\mathrm{HfO}_{2} \mathrm{O} \mathrm{K}_{1}$ spectra are of the order of $1 \mathrm{eV}$, and these have not been detected in the $\mathrm{Zr} \mathrm{M} 2,3$ spectrum where two peaks are resolved, or in the Hf $\mathrm{N}_{2,3}$ spectrum, where the crystal field splitting has not been resolved. A deconvolution of the $\mathrm{ZrO}_{2} 4 \mathrm{~d}$ features in the $\mathrm{ZrM}_{2,3}$ spectrum indicates a d-state splitting, $\Delta\left(\mathrm{d}_{1}, \mathrm{~d}_{2}\right)=2.2 \mathrm{eV}$, and the full-width at half-maximum (FWHM) for each of these features is $\sim 2.7 \mathrm{eV}$, which is well in excess of the spectral resolution of $<0.3 \mathrm{eV}$ of the measurement [6], [13]. Therefore the reason why term splittings are not observable in the $\mathrm{Zr} \mathrm{M}_{2,3}$ or $\mathrm{Hf} \mathrm{N}_{2,3}$ spectra is due to the short core life-time associated with the relatively high values of nuclear charge, 40 for $\mathrm{Zr}$ and 72 for Hf [12].

In marked contrast, the Jahn-Teller term splittings of for the Ti $3 \mathrm{~d}$ states are not observable in the $\mathrm{O} \mathrm{K}_{1}$ spectrum, but are easily resolved in the $\mathrm{Ti} \mathrm{L}_{3}$ spectrum. The ability to observe multiple features in $\mathrm{L}_{3}$ spectrum is a manifestation of a smaller value of $Z=22$, whereas the inability to observe multiple features in the $\mathrm{O} \mathrm{K}_{1}$ may be correlated with the large difference in matrix elements for the Jahn-Teller term split $\mathrm{T}_{2 \mathrm{~g}}$ states. It will demonstrated that these matrix element effects for the Jahn-Teller split $\mathrm{T}_{2 \mathrm{~g}}$ states are also found in the absorption edge spectrum as probed by photoconductivity [16], or spectroscopic ellipsometry [24].

\section{XAS Spectra of Complex Oxides}

The XAS spectra of stoichiometric $\mathrm{Zr}$ and $\mathrm{Hf}$ titanates exhibit Jahn-Teller term splittings in the $\mathrm{Ti}_{2,3}$ spectra, but none are detected in the respective $\mathrm{O} \mathrm{K}_{1}$ spectra. This is consistent with what has been observed for $\mathrm{TiO}_{2}$. In contrast $\mathrm{La}$ and other lanthanide RE scandates exhibit Jahn-Teller term splittings in the Sc $\mathrm{L}_{2,3}$ and $\mathrm{O} \mathrm{K}_{1}$ edge spectra, but not in the La, Dy or $\mathrm{Gd}_{2,3} \mathrm{M}_{2,3}$ spectra. These results suggest an empirical rule for Jahn-Teller term splittings in complex oxides that contain only $\mathrm{TM}$ and/or RE oxides. The bonding distortions that are favored by the Jahn-Teller effect occur in the atomic species with the lowest atomic d-state energy with respect to vacuum. Based on a computation by Harrision [14], the respective d-state energies of $\mathrm{Ti}, \mathrm{Zr}$ and $\mathrm{Hf}$ are $-11.05 \mathrm{eV},-8.46 \mathrm{eV}$ and $-8.14 \mathrm{eV}$, while those for: 1) Sc are $-9.35 \mathrm{eV}$, and 2) La, Gd and Dy, are approximately -6 to $-6.6 \mathrm{eV}$.

In the case of the scandates, the distortion also occurs at the atomic site with the lowest $\mathrm{O}$ atom coordination, six-fold coordinated Sc, and not at the site with the higher $\mathrm{O}$ atom coordination, twelve-fold coordinated La, Gd or Dy. Similar considerations based on relative d-state energies also apply to stoichiometric titanates in the binary oxide systems: $\mathrm{ZrO}_{2}-\mathrm{TiO}_{2}$ and $\mathrm{HfO}_{2}-\mathrm{TiO}_{2}$; however, there have been fewer studies of these binary oxide phase diagrams to identify all of the possible titanate phases, and in addition to determine the coordinations of the Ti and $\mathrm{Zr}$ atoms. In this regard, $\mathrm{Ti}$ in combination with O-atoms is usually six-fold coordinated, but $\mathrm{Zr}$ and $\mathrm{Hf}$ display both sixand eight-fold coordination as for example in $\mathrm{PbZr}(\mathrm{Hf}) \mathrm{O}_{3}$ and $\mathrm{Zr}(\mathrm{Hf}) \mathrm{O}_{2}$, respectively [4].

\section{Correlations Between XAS and Band Edge Spectra}

The term splittings in the $\mathrm{OK}_{1}$ spectra of $\mathrm{HfO}_{2}$ and $\mathrm{ZrO}_{2}$, and in the $\mathrm{L}_{3}$ spectrum of $\mathrm{TiO}_{2}$ have also been detected in the photoconductivity (PC) studies in [16], [26] and [27], and the vacuum ultra-violet spectroscopic ellipsomentry (VUV SE) studies of S. Zollner and co-workers [24], [25].

The majority of the spectral features attributed to band tail states in the PC and IPE spectra published in [16] and [26] are associated with band edge Jahn-Teller term split states discussed above. Fig. 16(a)-(d), are plots of the PC data for $\mathrm{ZrO}_{2}$, $\mathrm{HfO}_{2}, \mathrm{TiO}_{2}$ and $\mathrm{Al}_{2} \mathrm{O}_{3}$ in Fig. 1 of [16], that emphasize the band tail region. The band tail region of the $\mathrm{PC}$ spectrum of $\mathrm{Al}_{2} \mathrm{O}_{3}$ is also included in Fig. 16(d) for comparison.

The PC spectra for $\mathrm{ZrO}_{2}$ in Fig. 16(a) is for a thicker sample $(\sim 100 \mathrm{~nm})$ than that of $\mathrm{HfO}_{2}(\sim 20 \mathrm{~nm})$ and clearly reveals two photoconductivity edges, one at $\sim 5.6 \mathrm{eV}$ that defines the onset set of strong optical absorption that has been used to define the nominal bandgap of $\mathrm{ZrO}_{2}$, and second, much weaker edge at about $5.0 \mathrm{eV}$ that has not been assigned in any previous studies. This feature is also present in the VUV SE spectrum of $\mathrm{ZrO}_{2}$, and a specific structural/bonding assignment will be made in the next section of this paper.

The data for $\mathrm{HfO}_{2}$ in Fig. 16(b) also reveals two PC edges, one at $\sim 5.9 \mathrm{eV}$ that defines the onset of strong optical absorption that has been used to define the nominal bandgap of $\mathrm{HfO}_{2}$, 


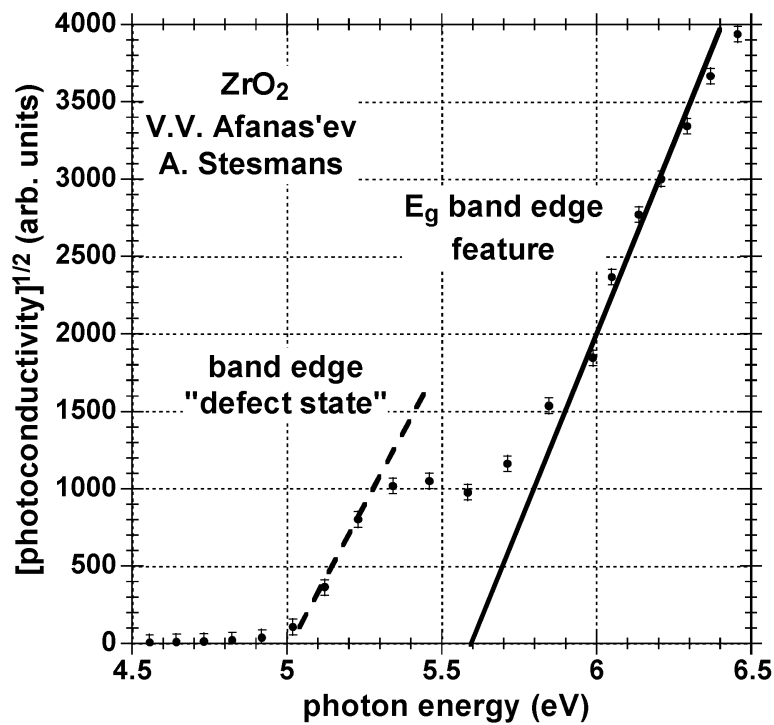

(a)

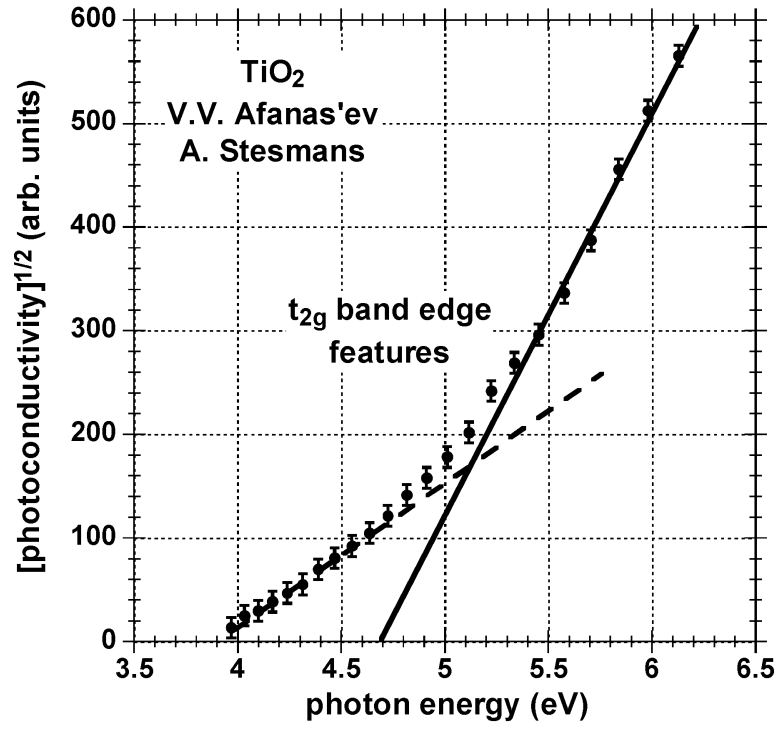

(c)

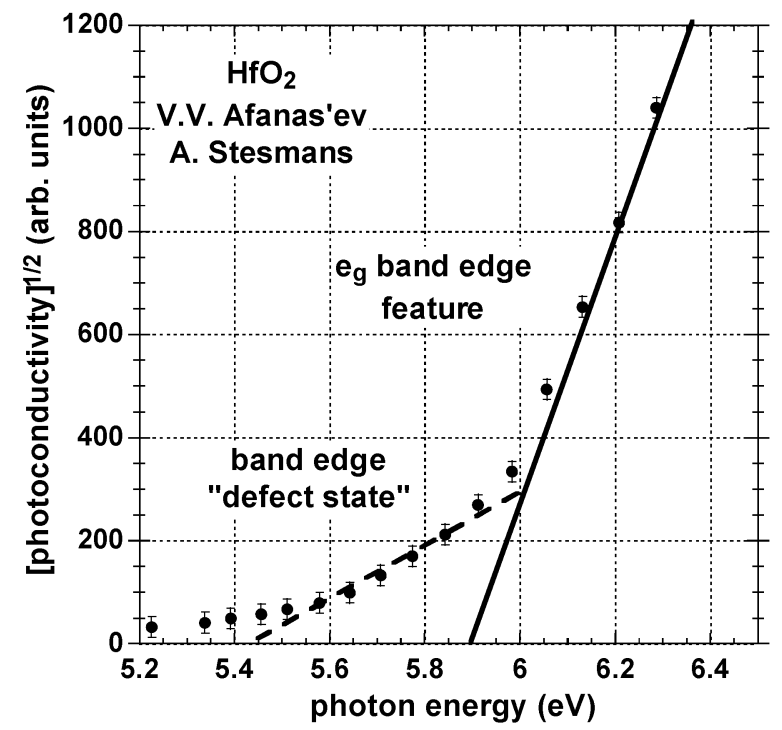

(b)

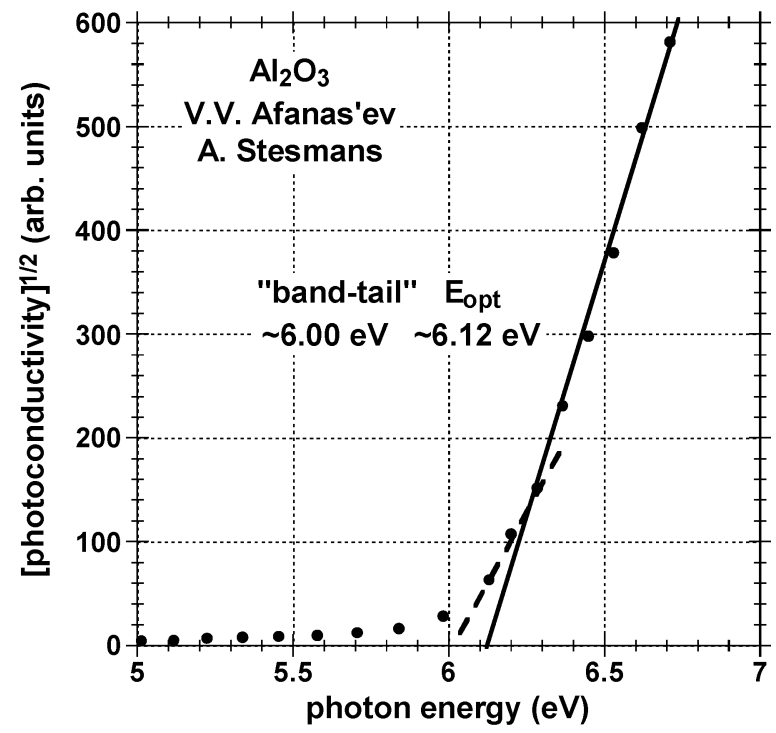

(d)

Fig. 16. Photoconductivity (PC) versus photon energy from [27] for (a) $\mathrm{ZrO}_{2}$, (b) $\mathrm{HfO}_{2}$, (c) $\mathrm{TiO}_{2}$ and (d) $\mathrm{Al}_{2} \mathrm{O}_{3}$ plotted to emphasize the weaker features (dashed lines) below the strong features (solid line) that define the onset of strong PC and optical absorption.

and second, significantly weaker edge at about $5.4 \mathrm{eV}$ that has not been assigned in any previous studies. Studies of the thickness dependence of the edge feature in $\mathrm{ZrO}_{2}$ are consistent with assigning the respective features in $\mathrm{ZrO}_{2}$ at $\sim 5.0 \mathrm{eV}$ and $\mathrm{HfO}_{2}$ at $\sim 5.45 \mathrm{eV}$ to the same microscopic "defect state." It is important to distinguish between band edge tail states that are due to disorder, as in the case on noncrystalline $\mathrm{Al}_{2} \mathrm{O}_{3}$ in Fig. 16(d), and localized defect states as in $\mathrm{ZrO}_{2}$ and $\mathrm{HfO}_{2}$ in Fig. 16(a) and (b), respectively. The extent of the disorder induced band tailing in $\mathrm{Al}_{2} \mathrm{O}_{3}$ is $\sim 0.15 \mathrm{eV}$, and the band edge defect states show a distinct edge behavior, particularly in the thicker $\mathrm{ZrO}_{2}$ film. This is evident in Fig. 2 of [27], which shows the PC of $\mathrm{ZrO}_{2}$ as a function of film thickness.

The data in Fig. 16(c) for $\mathrm{TiO}_{2}$ indicate two edges, one at $\sim 4.7 \mathrm{eV}$ that marks the onset of strong optical absorption, and another at $\sim 3.9 \mathrm{eV}$. The optical absorption at the band edge of $3.1 \mathrm{eV}$ is too weak to show up in these PC studies on thin film samples [15]. The two edges in this $\mathrm{PC}$ spectrum for $\mathrm{TiO}_{2}$ are assigned to intrinsic absorption states of $\mathrm{TiO}_{2}$; this assignment is supported by the VUV SE studies of the next section.

Finally, the PC studies of complex RE scandates in [26] display strong band tailing at energies below those generally assigned to the fundamental band edge. This traditional assignment of a bandgap is incorrect, and does not take into account strong matrix element effects for the band edge states of the RE scandates. This behavior for the scandates parallels the behavior for $\mathrm{TiO}_{2}$ and also has its origin in large differences matrix elements of Jahn-Teller term split states of octahedrally distorted bonding environments.

The PC spectrum of two representative scandate thin films, $\mathrm{LaScO}_{3}$ and $\mathrm{DyScO}_{3}$, are presented respectively in Fig. 17(a) and (b). Each of these films displays three quantitatively and qualitatively similar edge features at $\sim 5.6$ to $5.7 \mathrm{eV}, 5.1$ to 5.2 $\mathrm{eV}$ and $\sim 4.5 \mathrm{eV}$. Comparisons between these features and the 


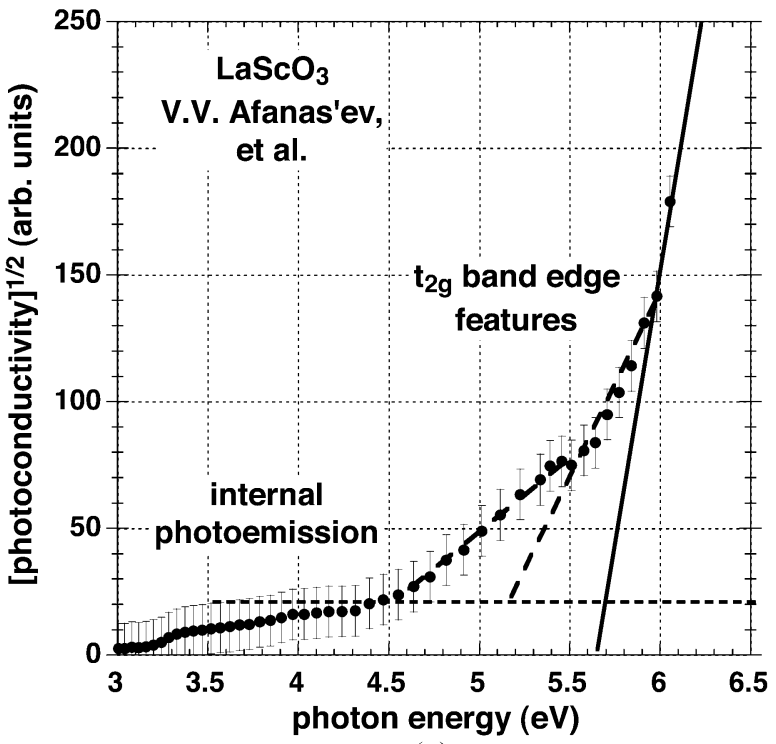

(a)

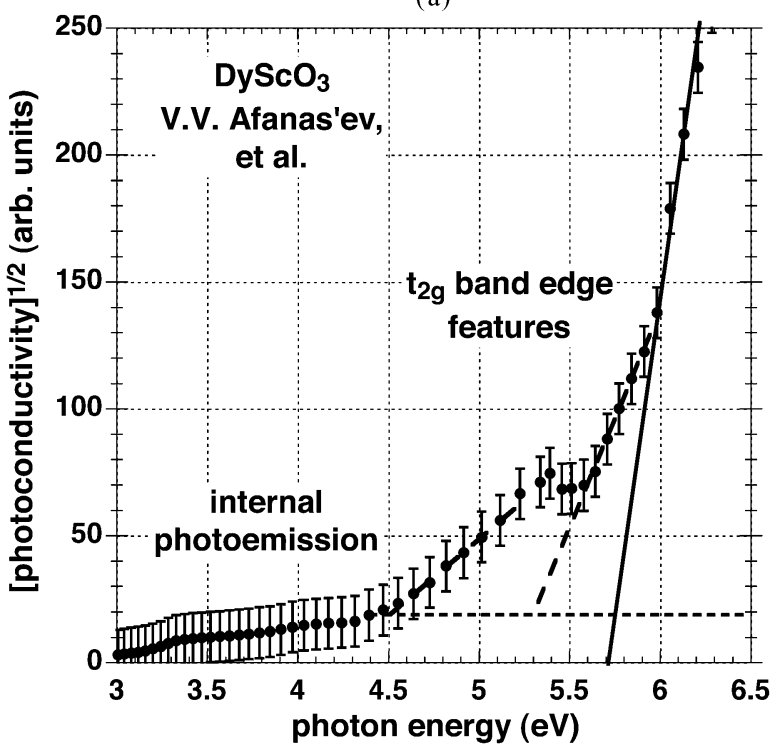

(b)

Fig. 17. Photoconductivity (PC) versus photon energy from [26] for (a) $\mathrm{LaScO}_{3}$ and (b) $\mathrm{DyScO}_{3}$ plotted to emphasize the weaker features (dashed lines) below the strong features (solid lines) that define the onset of strong PC and optical absorption.

term splittings of the $\mathrm{Sc}_{2 \mathrm{~g}}$ states that define the lowest conduction band edge indicate that they are intrinsic band edge states of the scandate complex oxides, and are transitions that originate in Sc $\pi$-bonding d-states in the valence band, and terminate in Sc $\pi$-anti-bonding $\mathrm{d}^{*}$-states of the conduction band. As noted above these $\mathrm{Sc} d$-states are coupled to $\mathrm{O} 2 \mathrm{p}$ states in the valence and $\mathrm{O} 2 \mathrm{p}^{*}$ states in the conduction band. In previously published papers, the strongest PC edge feature at $\sim 5.6$ to $5.7 \mathrm{eV}$ was used to define the conduction band edge in RE scandates. This assignment is simply incorrect. In this regard it is significant to note that the onset of strong absorption in crystalline $\mathrm{Sc}_{2} \mathrm{O}_{3}$ occurs at about $6 \mathrm{eV}$, but there is an absorption tail that extends to about $4.6 \mathrm{eV}$ [28]. Ref. [28] incorrectly defines the marked increase in absorption at $\sim 6 \mathrm{eV}$ as the $\mathrm{Sc}_{2} \mathrm{O}_{3}$ bandgap, ignoring the absorption tail that extends to about $4.6 \mathrm{eV}$, and failing to make comparisons with the well-understood absorption spectrum $\mathrm{TiO}_{2}$ [15].
The results presented in [9] for $\mathrm{GdScO}_{3}$ revealed an onset for strong absorption between 5.5 and $6 \mathrm{eV}$ as well as weaker absorption with a threshold extending to $\sim 4.5 \mathrm{eV}$. Therefore the results for the RE scandates and $\mathrm{Sc}_{2} \mathrm{O}_{3}$ support the interpretation presented above, that the d-state energies of same TM and RE atoms in elemental and complex oxides are at approximately the same relative energies with respect to vacuum, and that these energies correlate monotonically, and approximately linearly with the respective atomic d-state energies in the $\mathrm{s}^{2}$ atomic configuration tabulated by Harrison [14].

Fig. 18(a)-(c) display respectively the absorption constants, $\alpha$, for $\mathrm{TiO}_{2}, \mathrm{ZrO}_{2}$ and $\mathrm{HfO}_{2}$ nanocrystalline thin films calculated from the real and imaginary parts of the complex dielectric constant, $\varepsilon_{\mathrm{c}}=\varepsilon_{1}+\mathrm{i} \varepsilon_{2}$ [24], [25]. The values of $\varepsilon_{\mathrm{c}}$ were obtained from a fit to the optical constants obtained from spectroscopic ellipsometry data in the spectral range from approximately $1 \mathrm{eV}$ to $9.5 \mathrm{eV}$. The peaks in $\varepsilon_{2}$ better define the energies of the d-state features than do the relative maxima in $\alpha$, and these have been indicated in Fig. 18(a)-(c). The peaks in $\alpha$ are shifted in energy because they include a contribution from $\varepsilon_{1}$ as well as $\varepsilon_{2}$. Before commenting on the additional band edge feature in each of these spectra, it is important to note that there is excellent agreement between the relative energies of these features, and those obtained from either the $\mathrm{Ti}_{3}$ spectrum for $\mathrm{TiO}_{2}$, and the $\mathrm{O} \mathrm{K}_{1}$ spectra for $\mathrm{ZrO}_{2}$ and $\mathrm{HfO}_{2}$. This is illustrated in Fig. 19 in a plot that summarizes the energies of the Jahn-Teller term split d-state spectral features in the $\varepsilon_{2}$ spectrum of $\mathrm{TiO}_{2}$ as a function of the energies of the empty d-state features in the $\mathrm{Ti} \mathrm{L}_{3}$ spectrum. The slope of this plot is 1.1 establishing the correlation between the XAS results and the band edge absorption. A similar scaling holds for the d-state features in the $\varepsilon_{2}$ spectra of $\mathrm{ZrO}_{2}$ and $\mathrm{HfO}_{2}$, and the term splittings of the two $\mathrm{E}_{\mathrm{g}}$ and first $\mathrm{T}_{2 \mathrm{~g}}$ features in the respective $\mathrm{O} \mathrm{K}_{1}$ spectra.

The absorption spectra plots for $\mathrm{ZrO}_{2}$ and $\mathrm{HfO}_{2}$ in Fig. 18(b) and (c) respectively display an additional feature below the d-state features that have been correlated with XAS spectra and assigned to bulk nanocrystallite Jahn-Teller term split d-states. The additional feature is assumed to be an intrinsic defect state, i.e., one associated with the constituent atomic species of these elemental oxides. Based on the relatively high values of $\varepsilon_{2}$ and equivalently $\alpha$, for this feature, the concentration of these defects may be as high as $10^{20} \mathrm{~cm}^{-3}$. This assumption assumes an atomic absorption cross section/ $\mathrm{Zr}$ atom of $\sim 10^{-15}$ to $10^{-16} \mathrm{~cm}^{-2}$. This in turn corresponds to a bulk crystallite absorption coefficient between about $2 \times 10^{5}$ and $2 \times 10^{6} \mathrm{~cm}^{-1}$.

This high concentration of defects is consistent with grain-boundary defect states that derive from Jahn-Teller term splittings larger than those in the bulk of the nanocrystallites. In this model, the additional term split states result an increased bonding distortion at the $\mathrm{Zr} 14$ atomic sites at the grain boundary; e.g., 4-5 of the $\mathrm{O}$ nearest neighbors, and the second shell of $\mathrm{Zr}$ second neighbors may reside in one crystallite, and the remainder of the $\mathrm{O}$ and $\mathrm{Zr}$ neighbors in a second crystallite. Preliminary calculations based on $\mathrm{x}, \mathrm{y}$ and $\mathrm{z}$ directed distortions of $\mathrm{Ti}$ and $\mathrm{Zr}$ atoms from octahedral and cubic symmetry support this model. For the case Ti, changing the $z$ axis Ti-O bond length difference with respect to the in-plane bonding from a symmetric to an asymmetric distortion increases the 


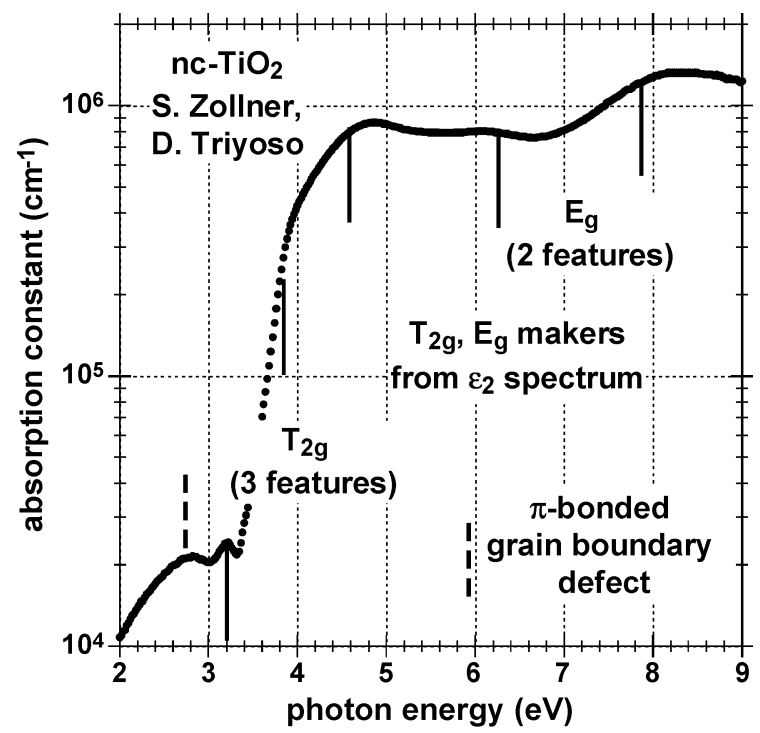

(a)

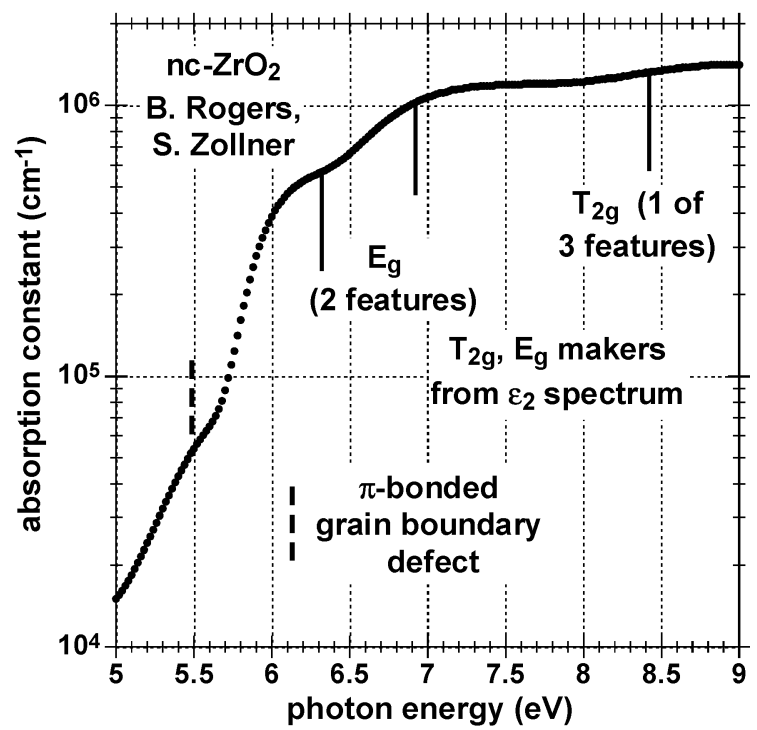

(b)

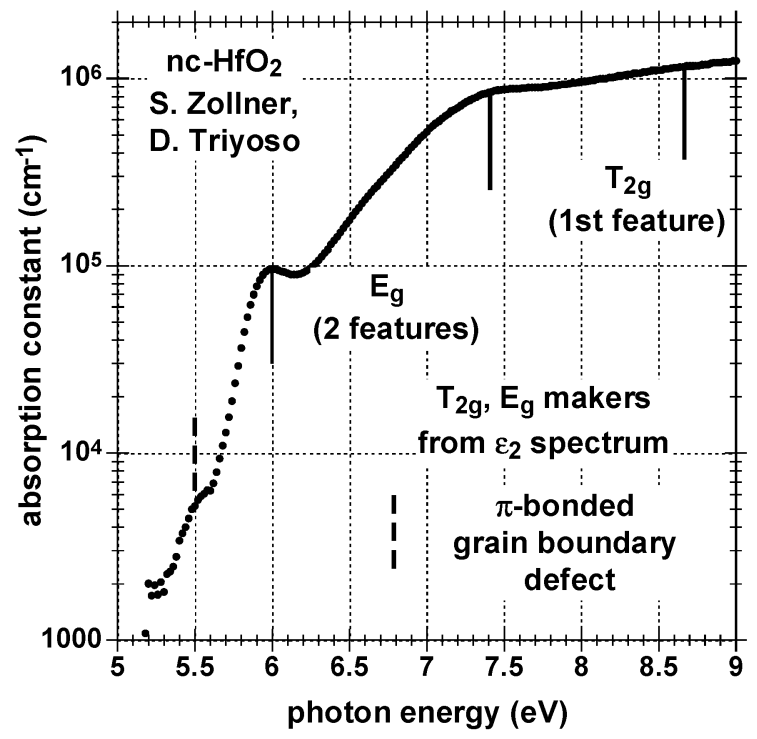

(c)

Fig. 18. Absorption constants, $\alpha$, versus photon energy of nanocrystalline thin films (a) $\mathrm{TiO}_{2}$, (b) $\mathrm{ZrO}_{2}$ and (c) $\mathrm{HfO}_{2}$ calculated from the real and imaginary parts of the complex dielectric constant, $\varepsilon_{\mathrm{c}}=\varepsilon_{1}+\mathrm{i} \varepsilon_{2}$, as obtained from spectroscopic ellipsometry studies. The solid markers indicate the energies of features in the respective $\varepsilon_{2}$ spectra, and the dashed markers indicate the defect state associated with grain boundaries.

term splitting of the lowest $\pi$-bonded d-state. The inclusion of these grain boundary Jahn-Teller distortions has been included in the expanded crystal-field/Jahn-Teller term splitting diagram in Fig. 20.

This assignment is also consistent with PC data from [27], which are presented in Fig. 16(a) for $\mathrm{ZrO}_{2}$ and (16b) for $\mathrm{HfO}_{2}$. These plots use the data in [27], but with increased emphasis on the photoconductivity beyond the primary absorption edge. These onset of strong PC in each of these thin film materials occurs at the same photon energies, $\sim 5.6$ in $\mathrm{ZrO}_{2}$ and $5.8 \mathrm{eV}$ in $\mathrm{HfO}_{2}$, as do the absorptions assigned to the lowest energy $\pi$-bonded $\mathrm{E}_{\mathrm{g}}$ state at the respective band edges. The additional PC response at significantly reduced levels extends to lower photon energies, $\sim 5.0 \mathrm{eV}$ for $\mathrm{ZrO}_{2}$ and $\sim 5.4 \mathrm{eV}$ for $\mathrm{HfO}_{2}$. These PC features are at approximately the same photon energy as the band edge defect states in the respective absorption constant spectra. However, the magnitude of the PC reduced by a larger factor. This is consistent with a hopping transport through localized states rather than a band-transport in extended states.

There is a significantly larger difference in the optical absorption of the lowest $\pi$-bonded $\mathrm{T}_{2 \mathrm{~g}}$ state in the absorption spectrum of $\mathrm{TiO}_{2}$ than in those of $\mathrm{ZrO}_{2}$ and $\mathrm{HfO}_{2}$. This low value of absorption makes it difficult, if not impossible, to observe PC below about $4 \mathrm{eV}$ in thin film $\mathrm{TiO}_{2}$. This is indeed the case, and the PC edge in this material displays two thresholds, one at $\sim 4.5$ $\mathrm{eV}$ and the section at $\sim 4 \mathrm{eV}$ that are assigned, respectively to the 2nd and 3rd $\mathrm{T}_{2 \mathrm{~g}} \pi$-bonded states of the absorption edge as display in Fig. 18(c).

Complex RE scandates display similar band edge spectral features. Comparisons between the published absorption edge data for $\mathrm{Sc}_{2} \mathrm{O}_{3}$ [28], previously published studies of complex oxides, and the PC edge spectra of [26], display essentially the same behavior for the TM oxides. These similarities include the following: 1) the lowest lying conduction band states in $\mathrm{Sc}_{2} \mathrm{O}_{3}$ 


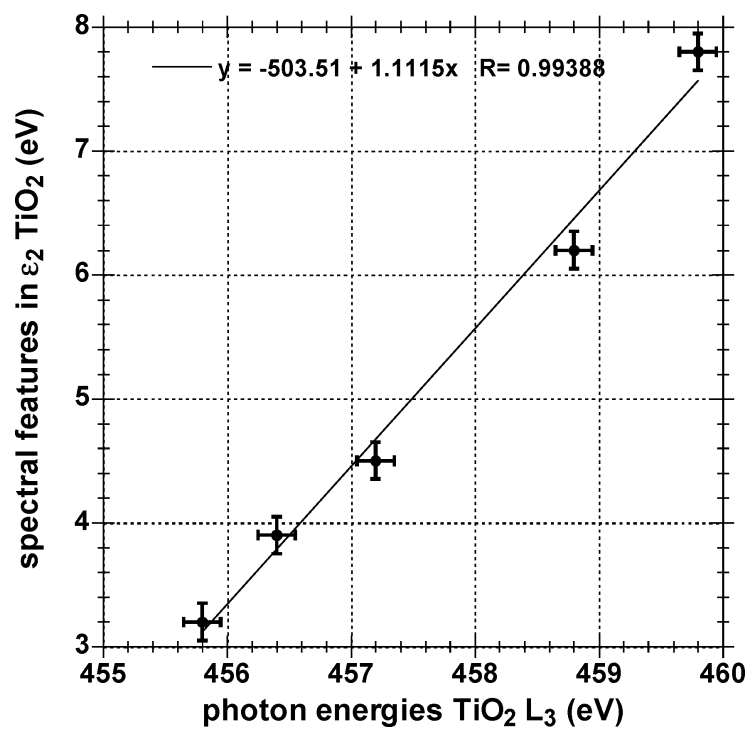

Fig. 19. Correlation between features of the $\mathrm{L}_{3}$ edge and $\varepsilon_{2}$ for $\mathrm{TiO}_{2}$.

from [28], and those revealed in XAS and PC spectra are approximately the same, with the effective band edge being determined by the lowest lying $\mathrm{T}_{2 \mathrm{~g}}$ term split state of $\mathrm{Sc}$ at about 4.6 $\mathrm{eV}$, and 2) the $\mathrm{Sc} \mathrm{T}_{2 \mathrm{~g}}$ state at $\sim 4.5-4.6 \mathrm{eV}$ displays approximately the same relative reductions in optical and $\mathrm{x}$-ray absorption strength as does the $\mathrm{Ti} \mathrm{T}_{2 \mathrm{~g}}$ state and $\mathrm{TiO}_{2}$ band edge absorption at $\sim 3.1 \mathrm{eV}[15]$.

PC spectra for representative RE scandates display essentially the same band edge, and band tail behavior as the TM oxides and this is illustrated in Fig. 17(a) for $\mathrm{LaScO}_{3}$ and Fig. 17(b) for $\mathrm{DyScO}_{3}$. The energies difference between the experimentally determined band edge states of $\mathrm{LaScO}_{3}$ and $\mathrm{TiO}_{2}$ is $\sim$ $1.6 \pm 0.2 \mathrm{eV}$. This energy difference is approximately equal to the difference of $\sim 1.7 \mathrm{eV}$ between their respective atomic state energies of -11.05 for $\mathrm{Ti}$ and -9.35 for Sc. The three PC thresholds at approximately $4.5 \mathrm{eV}, 5.0 \mathrm{eV}$ and $5.5 \mathrm{eV}$ are assigned the $\mathrm{Sc} \mathrm{T}_{2 \mathrm{~g}}$ states at the band edge, and the PC below $4.5 \mathrm{eV}$ is assigned to intrinsic defect states, some of which may be derived from Jahn-Teller term split states at internal grain boundaries.

Finally, similar Jahn-Teller term split features have been observed in $\mathrm{LaAlO}_{3}$. Band edge features are due to Jahn-Teller effect associated with three fold degenerate $\mathrm{Al} 3 \mathrm{p}$ states, whereas in, there is a crystal field splitting the La $5 \mathrm{~d}$ states as well. Consistent with a rhombohedral distortion in this perovskite the twelve-fold coordinated La atoms not longer reside in bonding site that complete spherical sysmmetry. This bonding is qualitatively different that the bonding of twelve fold coordinated Dy,Gd and La in the scandate perovskites that have an othrorhombic bonding distortion. This places the lowest bandgap of $\mathrm{LaAlO}_{3}$ at $\sim 4.6$ to $4.7 \mathrm{eV}$ consistent with the absorption spectrum presented in [9] for a relatively thick single crystal sample.

\section{Summary: Alternative High- $\kappa$ TM/RE GATE DIELECTRICS FOR CMOS DEVICES}

There are several important insights made in this paper relative to the application of elemental and complex TM/RE oxides, and silicate and aluminate alloys in advanced Si devices. These new insights into band edge properties play a significant role in limiting the field of alternative dielectrics that can be integrated into bulk CMOS devices to extend the life-time of this approach to memory and logic integrated circuits and systems. Additionally, these limitations apply to use of these materials with semiconductor substrates other than $\mathrm{Si}$ as well, including trained $\mathrm{Si}-\mathrm{Ge}, \mathrm{Ge}$ and compound semiconductor.

\section{A. Morphology of Deposited High- $\kappa$ Gate Dielectrics}

There has been much discussion about the character of deposited thin film dielectrics: are they amorphous, or nano- or polycrystalline? In a previous publication [29], one of the authors (GL) discussed a classification scheme based on bond-ionicity, and in the light of the spectroscopic studies reported in this review, it is important to review that approach, and indicate what has been learned in the years following the publication of that paper. The classification scheme was based on the well-established identification of three different bonding morphologies for glasses, and noncrsytalline solids in general: 1) continuous random networks, denoted here as CRNs; 2) disrupted or modified continuous random networks, denoted here as MCRNs; and 3 ) the random close packing of hard spheres, denoted here as RCPSs (random closed packed structures [30].

The prototypical CRN is noncrystalline $\mathrm{SiO}_{2}$, in the vitreous or glassy form, and additionally as thermally grown or deposited thin films as well. The bonding is predominantly covalent, or equivalently less than $50 \%$ ionic as in $\mathrm{SiO}_{2}$, where the Pauling bond ionicity, fi (or Ib) is 0.45 [29]. The bonding in these network structures follows the so-called 8-N rule [30], where $\mathrm{N}$ is the number of valence electrons. In $\mathrm{SiO}_{2}, \mathrm{~N}=4$ for $\mathrm{Si}$ and $\mathrm{N}=6$ for $\mathrm{O}$, so that the $\mathrm{Si}$ atoms are four-fold coordinated in a tetrahedral bonding arrangement, and the $\mathrm{O}$ atoms are two-fold coordinated, bridging the $\mathrm{Si}$ atoms with a $\mathrm{Si}-\mathrm{O}-\mathrm{Si}$ bond angle of $\sim 148$ degrees, and with a bond angle distribution of \pm 10 degrees. The dihedral angles between tetrahedral groups are random, and this disorder, combined with the large $\mathrm{Si}-\mathrm{O}-\mathrm{Si}$ bond angle distribution provides the configurational entropy that is important in promoting glass formation and/or noncrystallinity in thermally grown or deposited thin films.

Other CRNs include $\mathrm{SiO}_{2}$ solid solutions with boron oxide, $\mathrm{B}_{2} \mathrm{O}_{3}$ or phosphorous oxide, $\mathrm{P}_{2} \mathrm{O}_{5}$, silicon nitride, $\mathrm{Si}_{3} \mathrm{~N}_{4}$, and silicon oxynitride alloys, which are solid solutions of $\mathrm{SiO}_{2}$ and $\mathrm{Si}_{3} \mathrm{~N}_{4}$. The solid solution CRNs are differentiated from the MCRNs because each of the individual alloy constituents, e.g., $\mathrm{B}_{2} \mathrm{O}_{3}$ or $\mathrm{P}_{2} \mathrm{O}_{5}$, form $\mathrm{CRN}$ structures as well. The highest values of $\kappa$ attainable in low defect CRNs are approximately 5.5 and have been obtained in $\mathrm{Si}$ oxynitride alloys with approximately equal concentrations of $\mathrm{SiO}_{2}$ and $\mathrm{Si}_{3} \mathrm{~N}_{4}$ [31].

The prototypical MCRNs are silicates. These are also mixtures of oxides, but in this instance, the alloy constituents by themselves do not form CRN structures. Typical silicate alloys include mixtures of $\mathrm{SiO}_{2}$ and 1) sodium oxide, $\mathrm{Na}_{2} \mathrm{O} ; 2$ ) calcium oxide, $\mathrm{CaO}$; 3 ) magnesium oxide, $\mathrm{MgO}$; 4) $\mathrm{PbO}_{2}$, lead dioxide; and 5) $\mathrm{Al}_{2} \mathrm{O}_{3}$, aluminum oxide to name just a few of the many silicates that have been studied.

Each of these oxides has a significantly higher bond ionicity that $\mathrm{SiO}_{2}$, ranging from about 0.57 for $\mathrm{Al}_{2} \mathrm{O}_{3}$ to about 0.8 for 


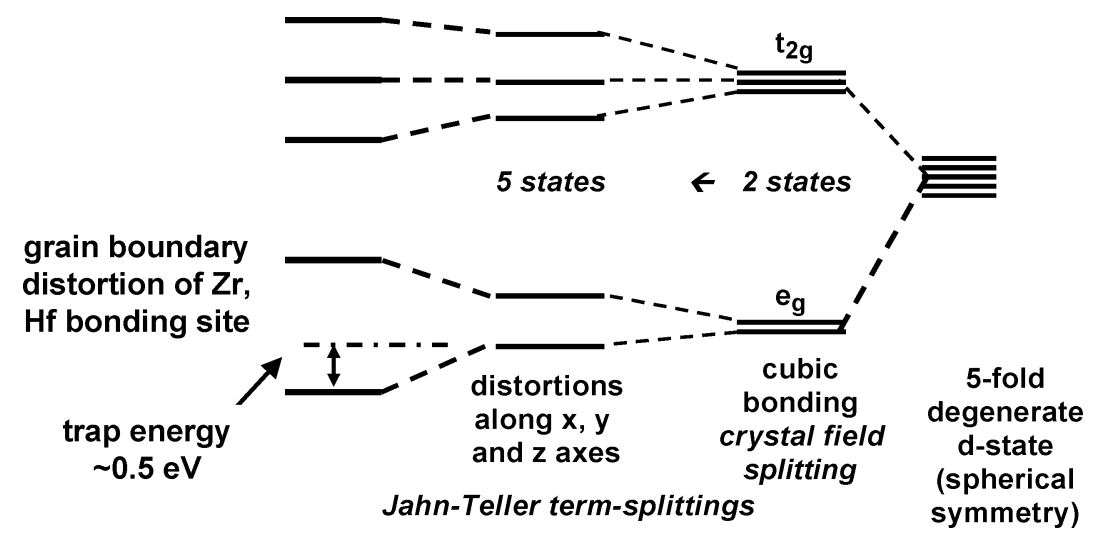

Fig. 20. Schematic representation of the Jahn-Teller distortions in TM elemental and complex oxides with eight-fold coordination, including the grain-boundary defect states.

$\mathrm{Na}_{2} \mathrm{O}$. The electro-positive atoms, $\mathrm{Na}, \mathrm{Ca}, \mathrm{Mg}$, etc., are incorporated in ionic bonding arrangements in which their bonding coordinations are the same as they are in the ionic constituent oxides, e.g., six for $\mathrm{Na}$ and $\mathrm{Ca}$. The bonding arrangements require the formation of electrostatic bonds that involve the nonbonding electrons on the bridging $\mathrm{O}$ atoms of the nondisrupted portion of the $\mathrm{SiO}_{2}$ network, thereby increasing the average coordination of these atoms from two to three. These arrangements therefore disrupt the CRN through the formation of ionic bonding arrangements, and modify the network through the increase in the coordination of the network oxygen atoms as well.

In addition to the silicates listed above, silicates can also be formed by combining $\mathrm{SiO}_{2}$ with TM oxides, such as $\mathrm{ZrO}_{2}$, $\mathrm{HfO}_{2}, \mathrm{Y}_{2} \mathrm{O}_{3}$ and $\mathrm{La}_{2} \mathrm{O}_{3}$, as well as trivalent rare earth atoms. Thin film TM and RE silicates have received attention as candidate materials for high- $\kappa$ applications. They suffer from one significant draw back that is common to all silicate alloys; they chemically phase separate at elevated temperatures, e.g., about $900{ }^{\circ} \mathrm{C}$, form two-phase mixtures of noncrystalline $\mathrm{SiO}_{2}$, and nano- or microcrystallites of the more ionic oxide component [32].

There is also a body of experimental results that suggests that $\mathrm{Al}_{2} \mathrm{O}_{3}$ and $\mathrm{Ta}_{2} \mathrm{O}_{5}$ form noncrystalline thin film solids when deposited or grown from the vapor phase. However, these materials as well crystallize at relatively low temperatures, 800 to $900{ }^{\circ} \mathrm{C}$ [32]. The highest values of obtained in these MCRNs are generally in the range of 10 , and in some instances up to about 15 .

The third class of noncrystalline materials are RCPSs, which consist primary of metal alloys such as $\mathrm{Gd}_{36} \mathrm{Fe}_{64}$ and $\mathrm{Tb}_{33} \mathrm{Fe}_{67}$, and metal-metalloid alloys such as $\mathrm{Ni}_{76} \mathrm{P}_{24}$ and $\mathrm{Co}_{78} \mathrm{P}_{22}$ [30], [33]. These alloys have been prepared by rapid quenching of liquids, and by vacuum deposition of thin films. These alloys exhibit a unique feature in the radial distribution functions (RDFs) obtained from Fourier transform the x-ray scattering intensity $(\mathrm{I}(\mathrm{k}))$ as a function of wave vector, $\mathrm{k}$. This is doublet in the second neighbor distance that corresponds to two different packings of hard spheres corresponding to: 1) in-line packing, in which two spheres of are each in direct contact with, and separated by a third sphere, and 2) a second packing arrangement in which the two spheres that separate a pair of spheres are in direct contact with each other and separate their two common neighbors. These two packing arrangements which can be permutted between the two components of a metallic alloy provide configuration entropy which contributes to glass formation.

The bond ionicity between the alloy components of these RCPSs is generally very small, less than $10 \%$ and does not mitigate the gains in the Gibbs free energy derived from the configuration entropy contributions.

One the other hand bond ionicities in excess of $65 \%$ for TM and RE oxides, and complex oxides make RCPSs energetically unfavorable because of Coulomb repulsions that completely mitigate the gains in configuration entropy form permutted packing arrangements such as those described above.

Therefore, as discussed above, when chemically pure, the morphology of TM and RE oxides, and TM and RE complex oxides will be crystalline. These materials are generally nanocrystalline as deposited, and crystallite sizes grow rapidly as annealing temperatures are increased above $700{ }^{\circ} \mathrm{C}$. If the crystallites are less than about $3 \mathrm{~nm}$ in average in as-deposited, these cannot be detected by x-ray diffraction (XRD), and these films have termed "x-ray amorphous". However, other techniques such as $\mathrm{x}$-ray absorption, as discussed above, can detect nanocrystallinity through Jahn-Teller term splittings.

\section{B. Band Gap and Conduction Band Offset Energy Scaling}

Band gap scaling has been addressed above in the context of the seminal papers of John Robertson that combine model calculations with a limited amount of experimental data [2]. The experimental results presented in this paper reinforce the conclusions addressed above, and discussed in other publications [6]. The conclusion is that the bandgaps of high- $\kappa \mathrm{TM} / \mathrm{RE}$ elemental oxides scale as the d-state energy of the respective TM/RE atoms. As noted in [6], this scaling may not apply to $\mathrm{Y}_{2} \mathrm{O}_{3}$ and $\mathrm{La}_{2} \mathrm{O}_{3}$ due to overlap with their respective $5 \mathrm{~s}$ and 6s states. However, the limitation discussed above remains the same: the bandgaps and conduction band offset energies of the elemental high- $\kappa$ dielectrics with highest static dielectric constants, $\mathrm{Sc}_{2} \mathrm{O}_{3}, \mathrm{TiO}_{2}, \mathrm{Ta}_{2} \mathrm{O}_{5}$ and $\mathrm{Nb}_{2} \mathrm{O}_{5}$ have bandgaps and conduction band offset energies too small for use as alternative gate dielectrics in advanced Si devices.

Based on comparisons between the XAS spectra and/or optical bandgaps of $\mathrm{TiO}_{2}$ and $\mathrm{Hf}$ and $\mathrm{Zr}$ titanate alloys, and $\mathrm{Sc}_{2} \mathrm{O}_{3}$ 
and RE scandate alloys, the Jahn-Teller term spittings, the optical bandgaps and therefore the conduction band offset energies for these nanocrystalline materials are essentially the same as those for $\mathrm{TiO}_{2}$ and $\mathrm{Sc}_{2} \mathrm{O}_{3}$ respectively, and therefore are too small for applications as alternative gate dielectrics in advanced Si devices.

Based on these scaling considerations, this restricts the field of high- $\kappa$ materials that have the potential for use as gate dielectrics in advanced $\mathrm{Si}$ devices to $\mathrm{Zr}, \mathrm{Hf}, \mathrm{Y}, \mathrm{La}$, and trivalent lanthanide rare earth oxides, and their respective silicate and aluminate alloys. However, two other issues must be addressed relative to these dielectrics: 1) the thermal stability of noncrystalline silicate and aluminate alloys, and 2) intrinsic defect states derived from grain boundaries in nanocrystalline thin films.

\section{Thermal Stability of Silicate and Aluminate Alloys}

The silicate and aluminate alloys of $\mathrm{Zr}$ and $\mathrm{Hf}$ are generally noncrystalline or amorphous as-deposited, but display chemical phase separation at temperatures of approximately $900{ }^{\circ} \mathrm{C}$. These result in the formation of a nanocrystalline $\mathrm{ZrO}_{2}$ or $\mathrm{HfO}_{2}$. This has two consequences: 1) in alloys with less than 50 molecular percent $\mathrm{ZrO}_{2}$ or $\mathrm{HfO}_{2}$, the diphasic character of the phase separated thin film materials reduces the static dielectric constants, and 2) in alloy with more than 50 molecular percent $\mathrm{ZrO}_{2}$ or $\mathrm{HfO}_{2}$, it leads to crystallization with internal grain boundaries. In either the case these phase-separated films do not meet electrical performance metrics for advanced Si devices.

Crystallization has been impeded by the incorporation of nitrogen in these films, however, many problems remain that related to process integration issues. These are beyond the scope of this paper and will not be addressed.

\section{Grain Boundary Defects and Electrical Performance}

Bias dependent trapping and trap-assisted direct tunneling have been reported in states conduction band edge states near the conduction band edge in stacks gate dielectrics comprised of a $\mathrm{Si}$ substrate, a thin $\mathrm{SiO}_{2}$ interfacial buffer layer, and a gate dielectric of $\mathrm{HfO}_{2}$ [20], [21]. The trap assisted tunnelling (TAT) has been reported for low levels of bias, $\sim 1 \mathrm{eV}$ above the flatband voltage, for both substrate and gate injection, while, the trapping or Frenkel Poole (FP) transport has been reported only for substrate injection. These results have correlated these bias dependent electrical properties with a high density of traps near the conduction band edge. Based on these studies, the energy of the trapping sites has been estimated at 0.5 to $0.8 \mathrm{eV}$ below the band edge for extended state transport. This estimated energy is in excellent agreement with the band edge defect state found in $\mathrm{PC}$ and absorption spectra, and assigned in this paper to grain boundary, Jahn-Teller term split states.

Other studies of trapping in $\mathrm{HfO}_{2}$ based dielectrics: 1) have found identified bias voltage level dependent effects in bias temperature instabilities (BTI) for substrate injection of electrons [34], that are also consistent with a high density of trapping states below the conduction band edge, and 2) have demonstrated that the traps at the conduction band edge of $\mathrm{HfO}_{2}$, estimated to be at a depth of 0.3 to $0.5 \mathrm{eV}$, are pre-existing and not generated as a result of carrier injection [20], [21], [35]. The densities of these band edge traps have been demonstrated to be at least $10^{12} \mathrm{~cm}^{2}$, and more likely in excess of $10^{13} \mathrm{~cm}^{3}$, corresponding to volume densities of at least $10^{18} \mathrm{~cm}^{-2}$, and probably as high as $3-5 \times 10^{19} \mathrm{~cm}^{-3}$. Based on the spectroscopic studies reported above, these trapping states are assigned to the states in $\mathrm{HfO}_{2}$ associated the spectral feature below the lowest energy Eg conduction band state, i.e., the state that extends to about $0.5 \mathrm{eV}$ below the conduction band edge. This defect has been assigned to a state resulting from a Jahn-Teller term split state that arises from a local bonding environment for $\mathrm{Hf}$ atoms at a grain boundary that is more distorted than at a $\mathrm{Hf}$ site in the bulk of a nanocrystallite. The density of these defects, as estimated from the optical absorption, and the PC is at least $10^{19} \mathrm{~cm}^{-2}$, and in the same general range as determined from the transport studies.

Similar band edge states have been found in $\mathrm{ZrO}_{2}$, and in the complex scandate dielectrics. Frenkel-Poole transport has been reported in $\mathrm{ZrO}_{2}$ dielectric films, and in $\mathrm{Ta}_{2} \mathrm{O}_{5}$ as well [1], [20], [21]. The spectroscopic studies of this paper suggest that bias-direction (substrate or gate) dependent trapping and/or trap assisted tunneling be studied in alternative gate dielectrics, particularly those that are known to be nanocrystalline.

In this regard, band edge trapping has not been reported in as-deposited $\mathrm{Zr}$ of $\mathrm{Hf}$ silicate alloys when these films have not been subjected to annealing that results in chemical phase separation and crystallization. This includes nitrided $\mathrm{Zr}$ and $\mathrm{Hf}$ silicate alloys. Nitridation of $\mathrm{HfO}_{2}$ provides improvements in carrier mobility, and these may be associated with preservation of a noncrystalline morphology which would eliminat grain-boundary defects that derive from increased Jahn-Teller bonding distortions.

\section{E. Where Do We Go From Here?}

There are other factors to address in the extension Si CMOS devices, as well as in devices based on other semiconductor substrates including $\mathrm{Si}-\mathrm{Ge}, \mathrm{Ge}$ and compound semiconductors. The results discussed above identify the importance of intrinsic defect states in nanocrystalline thin films and that can arise from Jahn-Teller term split states at the conduction band edge. There is an ongoing debate relative to the future of bulk CMOS, and a transition to semiconductor on insulator (SOI), and three-dimensional devices.

\section{REFERENCES}

[1] M. Houssa, High-k Gate Dielectrics, M. Houssa, Ed. Bristol, U.K.: Inst. Physics, 2004, ch. 1.1.

[2] J. Robertson, J. Vac. Sci. Technol., vol. B 18, p. 1785, 2000.

[3] F. A. Cotton and G. Wilkenson, Advanced Inorganic Chemistry, 3rd ed. New York: Interscience, 1972, ch. 20.

[4] H. Krebs, Fundamentals of Inorganic Crystal Chemistry. London, U.K.: McGraw-Hill, 1968, ch. 9 and 10.

[5] S. Okada and O. Matsuoka, J. Phys. Chem., vol. 91, p. 4193, 1989.

[6] G. Lucovsky, J. G. Hong, C. C. Fulton, Y. Zou, R. J. Nemanich, and H. Ade, J. Vac. Sci. Technol, vol. B 22, p. 2132, 2004.

[7] G. B. Rayner Jr., D. Kang, Y. Zhang, and G. Lucovsky, J. Vac. Sci. Technol., vol. B 20, p. 1748, 2002.

[8] C. C. Fulton, G. Lucovsky, and R. J. Nemanich, Appl. Phys. Lett., vol. 84 , p. 580, 2003.

[9] S. G. Lim et al., J Appl. Phys., vol. 91, pp. 4500-4505, 2002.

[10] L. F. Edge et al., Appl. Phys. Lett., vol. 86, p. 4629, 2004.

[11] Y. Zhang, Ph. D. Dissertation, Dept. Physics, North Carolina State Univ., Raleigh, 2005. 
[12] E. U. Condon and G. H. Shortley, The Theory of Atomic Spectra. Cambridge, U.K.: Cambridge Univ. Press, 1957, ch. V.

[13] G. Lucovsky et al., J. Vac. Sci. Technol., vol. B 20, p. 1739, 2002.

[14] W. A. Harrision, Elementary Electronic Structure, Singapore: World Scientific, 1999.

[15] P. A. Cox, Transition Metal Oxides. Oxford, U.K.: Oxford Science, 1992.

[16] V. V. Afanas'ev and A. Stesmans, High- $\kappa$ Gate Dielectrics, M. Houssa, Ed. Bristol, U.K.: Inst. Physics, 2004, ch. 3.3.

[17] International Technology Roadmap for Semiconductors (2001). [Online]. Available: http://public.itrs.net

[18] G. Lucovsky and J. C. Phillips, J. Vac. Sci. Technol., vol. B 22, p. 2089, 2004.

[19] G. Lucovsky, J. P. Maria, and J. C. Phillips, J. Vac. Sci. Technol., vol. B 22, p. 2097, 2004.

[20] J.-L. Autran, D. Munteanu, and M. Houssa, High- $\kappa$ Gate Dielectrics, M. Houssa, Ed. Bristol, U.K.: Inst. Physics, 2004, ch. 1.4.

[21] X. Zu, M. Houssa, S. De Gendt, and M. Hyens, Appl. Phys. Lett., vol. 80, p. 1975, 2001.

[22] L. F. Edge et al., presented at the Physics and Chemistry of Semiconductor Interfaces Conf., Bozman, MT, Jan. 2005.

[23] P. W. Peacock and J. Robertson, J. Appl. Phys., vol. 92, p. 4712, 2002.

[24] S. Zollner and D. Tyrioso, private communication.

[25] S. Zollner and B. R. Rogers, private communication.
[26] V. V. Afanas'ev et al., Appl. Phys. Lett., vol. 85, p. 5917, 2004.

[27] V. V. Afanas'ev and A. Stesmans, Appl. Phys. Lett., vol. 80, p. 1957, 2002.

[28] H. H. Tippins, J. Phys. Chem Solids, vol. 27, p. 1069, 1966.

[29] G. Lucovsky, J. Vac. Sci. Technol., vol. A 19, p. 1553, 2001.

[30] R. Zallen, The Physics of Amorphous Solids. New York: Wiley, 1983, ch. 1 and 2.

[31] G. Lucovsky, IBM J. Res. Dev., vol. 43, p. 301, 1999.

[32] G. Lucovsky, G. B. Rayner, and R. S. Johnson, Microelectron. Reliab., vol. 41, p. 937, 2001.

[33] C. S. Cargill III and S. Kirkpatrick, Structure and Excitations in Amorphous Solids, G. Lucovsky and F. L. Galeener, Eds. New York: American Inst. Physics, 1976, p. 339.

[34] J. Lee and K. Onishi, High- $\kappa$ Gate Dielectrics, M. Houssa, Ed. Bristol, U.K.: Inst. Physics, 2004, ch. 5.3.

[35] G. Bersuker et al., Microelectron. Reliabil., vol. 44, p. 1509, 2004.

Photographs and biographies of the authors not available at the time of publication. 